\title{
Performance Comparison of Watermarking Using SVD with Orthogonal Transforms and Their Wavelet Transforms
}

\author{
Dr. H. B. Kekre ${ }^{1}$ \\ ${ }^{1}$ Senior Professor, MPSTME, NMIMS University, Mumbai, India \\ E-mail: hbkekre@yahoo.com \\ Dr. Tanuja Sarode ${ }^{2}$ \\ ${ }^{2}$ Associate Professor, TSEC, University of Mumbai, Mumbai, India \\ E-mail: tanuja_0123@yahoo.com \\ Shachi Natu ${ }^{3}$ \\ ${ }^{3}$ Ph.D. Research Scholar, MPSTME, NMIMS University, Mumbai, India \\ E-mail: shachi_natu@yahoo.com
}

\begin{abstract}
A hybrid watermarking technique using Singular value Decomposition with orthogonal transforms like DCT, Haar, Walsh, Real Fourier Transform and Kekre transform is proposed in this paper. Later, SVD is combined with wavelet transforms generated from these orthogonal transforms. Singular values of watermark are embedded in middle frequency band of column/row transform of host image. Before embedding, Singular values are scaled with suitable scaling factor and are sorted. Column/row transform reduces the computational complexity to half and properties of singular value decomposition and transforms add to robustness. Behaviour of proposed method is evaluated against various attacks like compression, cropping, resizing, and noise addition. For majority of attacks wavelet transforms prove to be more robust than corresponding orthogonal transform from which it is generated.
\end{abstract}

Index Terms - Watermarking, Singular value Decomposition, wavelet transform, Kekre transform, Real Fourier Transform.

\section{INTRODUCTION}

In today's digital era, use of internet to disseminate digital images and other multimedia contents is inevitable. This imposes an immense need of security of digital contents transmitted over network. Availability of various tools and techniques allows easy manipulation of digital contents. To protect the digital contents from such undesirable alterations was the motivation for watermarking techniques. Though cryptographic techniques are there to provide security to digital contents, they don't contribute in protecting copyright of content owner. Watermarking techniques are explicitly meant for protecting the identity of owner of digital contents so that no one else can claim the ownership and can alter the contents.

While using Discrete Wavelet Transform, selection of appropriate frequency band plays an important role as it affects robustness and imperceptibility. Low frequency bands are normally major information contents of an image. Any modification to low frequency components therefore causes degradation into host image which is easily perceptible to Human Visual System [13]. However, in literature many methods of watermarking have been proposed which embed watermark in lower frequency components without losing imperceptibility of watermarked image. High frequency components in an image carry minimal information contents but they are responsible for edges in image. Since they carry minimum information about an image, alteration of these components due to embedding watermark is not easily sensed by human visual system. But it leads to high susceptibility to attacks like lossy image compression which eliminates high frequency components from image [13]. However it may prove more robust to other image processing attacks. To eliminate drawbacks of altering low frequency and high frequency components and to achieve benefits in terms of imperceptibility, selection of mid-frequency components is getting more attention in watermark embedding.

Remaining paper is organized as follows. Section II gives review of existing watermarking techniques in brief Section III briefly introduces wavelet transforms and singular value decomposition. Section IV describes proposed method in detail. Discussion regarding various attacks performed on watermarked images and response of proposed method to these attacks is given in section $\mathrm{V}$. Section VI compares performance of different column and row wavelet transforms separately. Section VII ends the paper with conclusion. 


\section{REVIEW OF LITERATURE}

In literature, various approaches have been tried out for digital watermarking using wavelet transform and singular value decomposition. Veysel Atlantas, A Latif Dogan, Serkan Ozturk [1] proposed a DWT-SVD based watermarking scheme using Particle Swarm Optimizer (PSO). Singular values of each sub-band of cover image are modified by different scaling factors. Modifications were further optimized using PSO to obtain highest possible robustness. Yang Qianli and Cai Yanhong [2] have proposed a DWT-DCT based watermarking wherein image is decomposed into its wavelet coefficients up to three levels. DCT of these coefficients is taken. Watermarking components are also transformed into DCT coefficients and then embedded into DCT coefficients of wavelet transformed image. Normalized Cross Correlation is used to detect the existence of watermark and PSNR is used to test the quality of watermarked image. In a watermarking method given by Xi-Ping $\mathrm{He}$ and Qing-Sheng Zhu [3], the wavelet transform is applied to local sub-blocks of image extracted randomly. Watermark image is then adaptively embedded into part of the sub-band coefficients by computing their statistical characteristics. SVD-DCT based watermarking technique is proposed by Zhen Li, Kim-Hui Yap and Bai-Ying Lei [4]. In this technique first SVD of image blocks is computed. Then first few singular values are selected and DCT is applied to them. High frequency band from this SVD-DCT block is selected for watermark embedding. In [5], Rahim Ansari, Mrutyunjaya M Devanalamath, K. Manikantan, S. Ramachandran, proposed a Digital Watermarking Algorithm using a unique combination of Discrete Wavelet Transform (DWT), Discrete Fourier Transform (DFT) and Singular Value Decomposition (SVD) for secured transmission of data through watermarking digital colour images. The singular values obtained from SVD of DWT and DFT transformed watermark is embedded onto the singular values obtained from SVD of DWT and DFT transformed colour image. Scaling and shift invariance property of DFT, rotation invariance property of SVD and robustness of DWT to compression are used to perform secure transmission of data through watermarking. Zhen Li, Kim-Hui Yap and Bai-Ying Lei have proposed a SVD-DCT based watermarking method in [6]. After applying SVD to the cover image blocks, DCT on the macro block comprised of the first singular values of each image block is taken. Watermark is embedded in the high-frequency band of the SVD-DCT block by imposing a particular relationship between some pseudo-randomly selected pairs of the DCT coefficients. Yan Dejun, Yang Rijing, Li Hongyan, and Zheng Jiangchao in [7] proposed a robust digital image watermarking technique based on Singular Value Decomposition (SVD) and Discrete Wavelet Transform (DWT). Spatial relationship of visually recognizable watermark is scattered using Arnold transform. Further, security is enhanced by performing chaotic encryption using chaotic Logistic Mapping. Host image is decomposed into four frequency bands using wavelet decomposition. LL frequency band is decomposed into non-overlapping $4 \times 4$ blocks and SVD is applied to each block. Largest singular value of each block is modified with the help of watermark. Inverse SVD followed by inverse DWT is applied to get watermarked image. Reverse steps are followed to recover the watermark from watermarked image. PSNR and Normalized Cross Correlation (NCC) are the metrics used to measure imperceptibility and robustness of the technique.

\section{WAVELET TRANSFORMS AND SINGULAR VALUE DECOMPOSITION (SVD)}

Instead of using traditional Haar wavelet, wavelet transforms are generated from orthogonal transforms using a new wavelet generation algorithm proposed by Dr. Kekre in [11]. These transforms can be generated using different possible size combinations of orthogonal transforms. Required global or local properties of component transforms can be varied by changing the size of component matrix. The concept can be extended further for generation of hybrid wavelet transforms which are composed of two different orthogonal transforms [15].

In past few years, wavelet transforms and SVD are being widely used for many image processing applications including digital watermarking.

Using singular value decomposition, any real matrix A can be decomposed into a product of three matrices $\mathrm{U}, \mathrm{S}$ and $\mathrm{V}$ as $\mathrm{A}=\mathrm{USV}^{\mathrm{T}}$, where $\mathrm{U}$ and $\mathrm{V}$ are orthogonal matrices and $\mathrm{S}$ is diagonal matrix. If $\mathrm{A}$ is mxn matrix, $\mathrm{U}$ is mxm orthonormal matrix whose columns are called as left singular vectors of $\mathrm{A}$ and $\mathrm{V}$ is $\mathrm{nxn}$ orthonormal matrix whose columns are called right singular vectors of A [8].

Some properties of SVD which make it useful in image processing are:

- The singular values are unique for a given matrix.

- The rank of matrix A is equal to its nonzero singular values. In many applications, the singular values of a matrix decrease quickly with increasing rank. This property allows us to reduce the noise or compress the matrix data by eliminating the small singular values or the higher ranks [9].

- The singular values of an image have very good stability i.e. when a small perturbation is added to an image; its singular values don't change significantly [10].

\section{PROPOSED METHOD}

Orthogonal transforms like DCT, Walsh, Haar, DKT and Real Fourier transform and their wavelets are used. Transform is applied to each plane of host image. Instead of full transform only column or row transform of image is taken. Column transform of image matrix $\mathrm{f}$ is given by (1) below where $\mathrm{f}$ is image to be transformed, $\mathrm{T}$ is transform matrix and $\mathrm{F}$ is transformed image. 


$$
[\mathrm{F}]=[\mathrm{T}] *[\mathrm{f}]
$$

Inverse column transform is given by (2).

$$
\mathrm{f}=[\mathrm{T}]^{\prime *}[\mathrm{~F}]
$$

Similarly row transform and inverse row transform are given by (3) and (4) respectively.

$$
\begin{aligned}
& {[\mathrm{F}]=[\mathrm{f}] *[\mathrm{~T}]} \\
& {[\mathrm{f}]=[\mathrm{F}] *[\mathrm{~T}]}
\end{aligned}
$$

Thus column or row transform reduces the number of computations to half of those in full transform.

After taking column transform, tendency of image energy concentration is observed to be towards upper rows and in case of row transform, it is observed to be towards left columns of an image. To achieve invisibility and robustness is the challenge in watermarking as they have trade-off. Thus higher robustness may lead to lower imperceptibility of watermarked image. Hence selection of moderate frequency components is required. In full transform, HL and LH frequency bands can be selected for the purpose. However this is not possible for column and row transform. Thus for column transform, moderate frequency elements are in the middle few rows of an image and for row transform it is in middle columns. Hence proper selection of rows in column transform and proper section of columns in row transform is necessary to achieve higher robustness and imperceptibility. For the proposed method, we tried many different ranges of middle frequency elements and ended with the frequency band from row 101 to 130. Singular value decomposition of watermark is obtained. Due to high energy compaction in SVD, we can choose only first few singular values to embed the watermark. This reduces the payload of information to be embedded and also increases imperceptibility. In proposed method first 30 singular values are selected. These values correspond to $99.1944 \%$ of watermark energy embedded in host. The middle frequency band elements of host are sorted in descending order. Singular values are scaled down and embedded in such a way that first two values are placed at the positions where energy gap between these values and corresponding frequency elements of host is the minimum. Remaining singular values are placed in consecutive positions after the position of second singular value in host. Scaling factor selection is done adaptively so that first singular value to be embedded maps exactly to highest middle frequency element from the selected band. Inverse column/ row transform of host after substituting singular values yields watermarked image.

Extraction is followed by reversing the steps of embedding. Here first column/ row transform of watermarked image is obtained. From this transformed image middle frequency elements are extracted from the positions where they were embedded. These elements contain the watermark in the form of scaled down singular values. Extracted elements are scaled up and are used to reconstruct the watermark. Quality of recovered watermark is compared with that of embedded watermark by calculating mean absolute pixel difference or Mean Absolute Error between them. Also quality of watermarked image is compared with quality of host image by calculating MAE between the two.

Fig. 1 shows the set of host images and watermark used for experimental work.

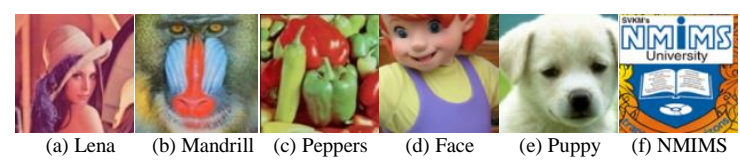

Fig. 1. (a)-(e) $256 \times 256$ size host images, (f) 128x128 size watermark used for experimental work.

In the proposed watermarking technique, wavelet transforms are generated from corresponding orthogonal transforms using Kekre's wavelet generation algorithm proposed in [11]. Since we need 256x256 size transform matrix for host, according to the algorithm in [11], we can have following combinations of component orthogonal transforms: $(128,2),(64,4),(32,8),(16,16)$, $(8,32),(4,64),(2,128)$ to generate required size transform matrix. For the proposed work the combinations selected for generation of wavelet transform are $(64,4),(32,8)$, $(16,16),(8,32),(4,64)$.

\section{PERFORMANCE OF PROPOSED TECHNIQUE AGAINST VARIOUS ATTACKS}

To verify the robustness of proposed watermarking technique, watermarked images are subjected to attacks like compression, cropping, noise addition, resizing and histogram equalization. MAE between the embedded watermark and watermark extracted from attacked image measures the robustness. In following subsections each of the attack with variations into it and their results are shown. Representative results of each attack are shown using image Lena for DCT and DCT wavelet (column and row version) and performance of each transform is shown in tables by taking average value of MAE of five host images.

\section{A. COMPRESSION OF WATERMARKED IMAGES}

Since limited bandwidth is to be used efficiently for data transmission, compression of data with minimum or acceptable level of information loss is the trend. It applies to watermarked images as well. Here watermarked images are compressed in three ways (a) using different orthogonal transforms (DCT, DST, Walsh, Haar and DCT wavelet), (b) using JPEG compression and (c) using Vector Quantization.

Results of these compression attacks are shown in Fig. 2. For each recovered watermark image MAE and for each wavelet transforms (column and row) the combination of component transform which gives minimum MAE is mentioned below the image.

Result images for Compression using Walsh are shown in Fig. 2 below. 


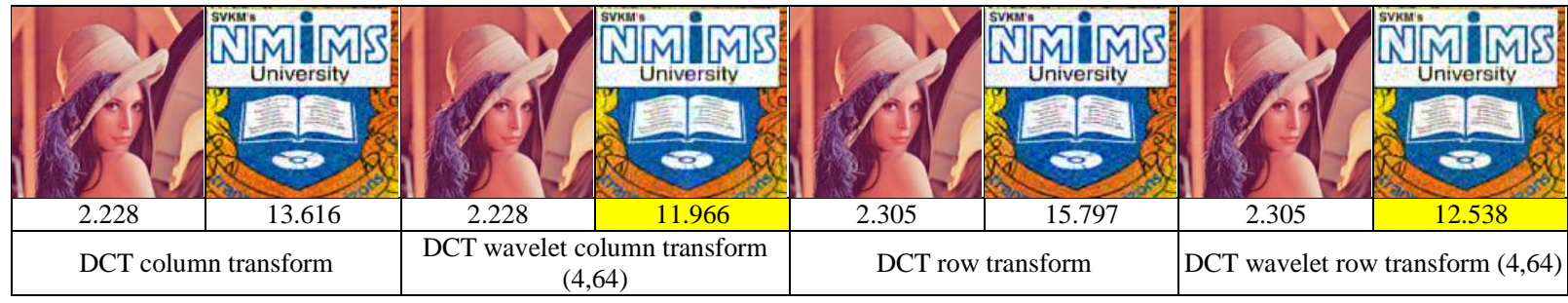

Fig. 2. result images for compression using Walsh transform when DCT column, DCT wavelet column, DCT row and DCT wavelet row transform is used to embed watermark

From Fig. 2 it can be seen that, DCT wavelet transform obtained from $(4,64)$ combination of component transforms (DCT) gives better quality of recovered watermark than DCT. It has been observed that for other transform based compressions except using DCT, DCT wavelet is better in robustness than DCT. Table 1 below summarizes the results of transform based compression attack for DCT and DCT wavelet transform.

Table 1. MAE between embedded and extracted watermark against transform based compression attack when DCT column and DCT wavelet column transform are used for embedding

\begin{tabular}{|c|c|c|c|c|c|c|}
\hline Transform used for compression Attack & DCT column transform & \multicolumn{4}{|c|}{ DCT wavelet column transform combinations } \\
\hline & & $\mathbf{( 1 6 , 1 6 )}$ & $\mathbf{( 3 2 , 8 )}$ & $\mathbf{( 8 , 3 2})$ & $\mathbf{( 6 4 , 4 )}$ & $\mathbf{( 4 , 6 4 )}$ \\
\hline DCT & 0.000 & 6.958 & 9.875 & 5.150 & 12.661 & $\mathbf{3 . 3 1 0}$ \\
\hline DST & 0.516 & 6.928 & 9.772 & 5.135 & 12.941 & $\mathbf{3 . 2 9 9}$ \\
\hline Walsh & 13.616 & 16.840 & 22.600 & 12.630 & 17.373 & $\mathbf{1 1 . 9 6 6}$ \\
\hline Haar & 45.764 & 36.397 & 17.214 & 49.284 & $\mathbf{1 7 . 1 1 4}$ & 17.821 \\
\hline DCT wavelet & 58.686 & $\mathbf{0 . 0 0 0}$ & 31.713 & 50.222 & 34.372 & 42.518 \\
\hline
\end{tabular}

From Table 1, it is clear that MAE between embedded and extracted watermark given by column DCT wavelet for Haar and DCT wavelet based compression are far better than column DCT. For other transforms used for compression, DCT wavelet gives acceptable MAE values.
The smallest MAE values given by column DCT wavelet are highlighted.

Table 2 shows MAE values between embedded and extracted watermark for transform based compression attack using DCT row and DCT wavelet row transform for embedding.

Table 2. MAE between embedded and extracted watermark against transform based compression attack when DCT row and DCT wavelet row transform are used for embedding

\begin{tabular}{|c|c|c|c|c|c|c|}
\hline Transform used for compression Attack & DCT row transform & \multicolumn{4}{|c|}{ DCT wavelet row transform combinations } \\
\hline & & $\mathbf{( 1 6 , 1 6 )}$ & $\mathbf{( 3 2 , 8 )}$ & $\mathbf{( 8 , 3 2 )}$ & $\mathbf{( 6 4 , 4 )}$ & $\mathbf{( 4 , 6 4 )}$ \\
\hline DCT & 0.000 & 6.485 & 9.029 & 4.637 & 12.259 & $\mathbf{2 . 9 6 0}$ \\
\hline DST & 0.509 & 6.509 & 8.999 & 4.655 & 12.695 & $\mathbf{3 . 0 3 5}$ \\
\hline Walsh & 15.797 & 16.707 & 20.532 & 13.574 & 15.259 & $\mathbf{1 2 . 5 2 8}$ \\
\hline Haar & 50.709 & 27.361 & 32.886 & 24.698 & $\mathbf{2 0 . 7 9 2}$ & 33.989 \\
\hline DCT wavelet & 70.760 & $\mathbf{0 . 0 0 0}$ & 26.201 & 28.597 & 27.538 & 51.150 \\
\hline
\end{tabular}

From Table 2, it is observed that use of DCT wavelet row transform for embedding watermark shows significant performance improvement for compression using Haar and DCT wavelet over row DCT used for embedding.

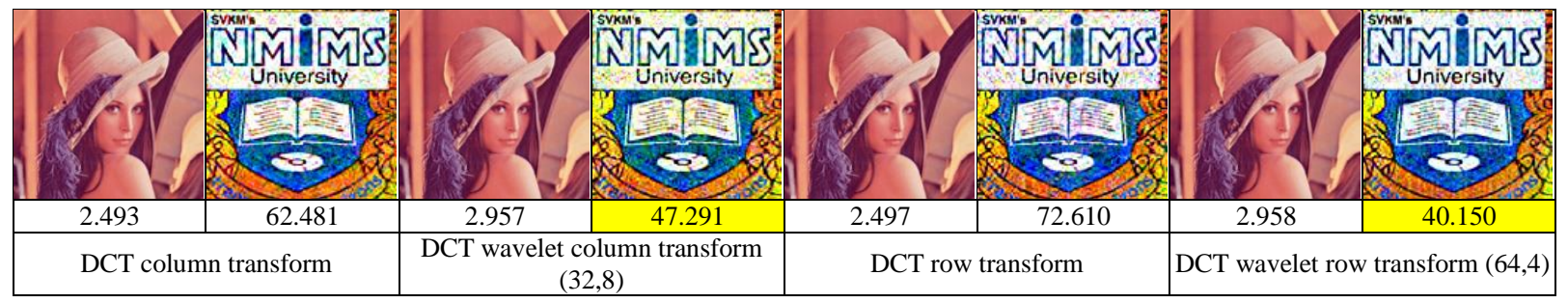

Fig. 3. Result images for VQ compression attack when DCT column and DCT wavelet column transform

From Fig. 3, it can be observed that wavelet transform (column or row version) gives better robustness than its component orthogonal transform for compression attack using Vector Quantization. Similar observations are noted
Fig. 3 shows the watermark extracted using DCT column and DCT wavelet column from VQ compression attack. for JPEG compression attack also. MAE values between embedded and extracted watermark obtained for VQ compression and JPEG compression using DCT column transform and various combinations of DCT wavelet 
column transform are shown in Table 3. Minimum values

given by wavelet transform combination are highlighted.

Table 3. MAE between embedded and extracted watermark against VQ based compression and JPEG compression using DCT column transform and DCT wavelet column transform

\begin{tabular}{|c|c|c|c|c|c|c|}
\hline Type of compression Attack & DCT column transform & \multicolumn{5}{|c|}{ DCT wavelet column transform combinations } \\
\hline & & $(16,16)$ & $(32,8)$ & $(8,32)$ & $(64,4)$ & $(4,64)$ \\
\hline Vector Quantization & 62.481 & 58.214 & $\mathbf{4 7 . 2 9 1}$ & 61.245 & 47.701 & 59.080 \\
\hline JPEG compression & 83.452 & 69.599 & 64.548 & 67.406 & $\mathbf{6 0 . 6 2 4}$ & 73.487 \\
\hline
\end{tabular}

Table 4 gives the MAE values between embedded and extracted watermark for VQ compression and JPEG compression attack using row versions of DCT and DCT wavelet.

Table 4. MAE between embedded and extracted watermark against VQ based compression and JPEG compression using DCT row transform and DCT wavelet row transform

\begin{tabular}{|c|c|c|c|c|c|c|}
\hline Type of compression Attack & DCT row transform & \multicolumn{4}{|c|}{ DCT wavelet row transform combinations } \\
\hline & & $(16,16)$ & $(32,8)$ & $(8,32)$ & $(64,4)$ & $(4,64)$ \\
\hline Vector Quantization & 72.610 & 59.801 & 49.102 & 61.160 & $\mathbf{4 0 . 1 5 0}$ & 62.164 \\
\hline JPEG compression & 84.252 & 69.099 & 69.111 & 71.109 & $\mathbf{6 2 . 6 2 9}$ & 76.094 \\
\hline
\end{tabular}

Behavior of other column transforms namely Haar, Walsh, Kekre transform and Real Fourier transform and their wavelet transforms against compression attack is shown in Table 5 to Table 8. Cells highlighted in yellow color represent the minimum MAE obtained by wavelet transform. Cells highlighted in blue color represent that orthogonal transform gives smaller error than wavelet transform. However, such occurrences are very less as can be seen from Table 5 to Table 8 . Hence we can conclude that column wavelet transforms are more robust than respective column orthogonal transforms against compression attack performed in various ways.

Table 5. MAE between embedded and extracted watermark against compression using Haar column transform and Haar wavelet column transform

\begin{tabular}{|c|c|c|c|c|c|c|}
\hline \multirow{2}{*}{ Compression using } & \multicolumn{5}{|c|}{ Haar wavelet column transform } & \multirow{2}{*}{ Column Haar } \\
\cline { 2 - 7 } & $\mathbf{( 1 6 , 1 6 )}$ & $\mathbf{( 3 2 , 8 )}$ & $\mathbf{( 8 , 3 2 )}$ & $\mathbf{( 6 4 , 4 )}$ & $\mathbf{( 4 , 6 4 )}$ & \\
\hline DCT & $\mathbf{2 . 7 4 2}$ & 4.283 & 2.786 & 4.036 & 3.206 & 3.480 \\
\hline DST & 2.881 & 4.288 & $\mathbf{2 . 8 7 8}$ & 3.825 & 3.438 & 4.054 \\
\hline Walsh & 3.557 & 4.855 & $\mathbf{2 . 6 8 2}$ & 8.337 & 4.291 & 8.429 \\
\hline M Haar & 0.000 & 0.000 & 0.000 & 0.000 & 0.000 & 0.000 \\
\hline JPEG & 58.148 & $\mathbf{5 6 . 8 0 4}$ & 58.843 & 59.828 & 57.946 & 61.223 \\
\hline VQ compression & $\mathbf{3 9 . 2 6 0}$ & 40.194 & 41.140 & 50.400 & 45.926 & 48.788 \\
\hline DCT wavelet & 44.043 & 47.070 & $\mathbf{4 3 . 4 5 8}$ & 43.511 & 45.817 & 44.373 \\
\hline
\end{tabular}

Table 6. MAE between embedded and extracted watermark against compression using Walsh column transform and Walsh wavelet column transform

\begin{tabular}{|c|c|c|c|c|c|c|}
\hline \multirow{2}{*}{ Compression using } & \multicolumn{5}{|c|}{ Walsh wavelet column transform } & \multirow{2}{*}{ Column Walsh } \\
\cline { 2 - 6 } & $\mathbf{( 1 6 , 1 6 )}$ & $\mathbf{( 3 2 , 8 )}$ & $\mathbf{( 8 , 3 2 )}$ & $\mathbf{( 6 4 , 4 )}$ & $\mathbf{( 4 , 6 4 )}$ & \\
\hline DCT & 1.652 & 2.566 & $\mathbf{1 . 4 4 0}$ & 1.886 & 1.886 & 1.377 \\
\hline DST & 1.700 & 2.877 & $\mathbf{1 . 4 9 7}$ & 2.025 & 2.025 & 1.458 \\
\hline Walsh & $\mathbf{0 . 0 0 0}$ & 4.215 & $\mathbf{0 . 0 0 0}$ & 1.405 & 1.405 & 0.000 \\
\hline M Haar & $\mathbf{0 . 0 0 0}$ & $\mathbf{0 . 0 0 0}$ & $\mathbf{0 . 0 0 0}$ & $\mathbf{0 . 0 0 0}$ & $\mathbf{0 . 0 0 0}$ & 7.084 \\
\hline JPEG & 63.002 & $\mathbf{6 2 . 1 1 2}$ & 63.491 & 62.868 & 62.868 & 71.660 \\
\hline VQ compression & $\mathbf{4 5 . 6 5 6}$ & 47.222 & 54.683 & 49.187 & 49.187 & 60.031 \\
\hline DCT wavelet & 54.575 & $\mathbf{5 4 . 5 7 5}$ & 56.897 & 55.349 & 55.349 & 60.842 \\
\hline
\end{tabular}

Table 7. MAE between embedded and extracted watermark against compression using Kekre column transform and Kekre wavelet column transform

\begin{tabular}{|c|c|c|c|c|c|c|}
\hline \multirow{2}{*}{ Compression using } & \multicolumn{5}{|c|}{ Kekre wavelet column transform } & \multirow{2}{*}{ Column Kekre Transform } \\
\cline { 2 - 6 } & $\mathbf{( 1 6 , 1 6 )}$ & $\mathbf{( 3 2 , 8 )}$ & $\mathbf{( 8 , 3 2 )}$ & $\mathbf{( 6 4 , 4 )}$ & $\mathbf{( 4 , 6 4 )}$ & \\
\hline DCT & $\mathbf{1 8 . 3 4 0}$ & 24.493 & 20.414 & 23.056 & 19.610 & 20.432 \\
\hline DST & $\mathbf{1 8 . 2 2 3}$ & 24.534 & 20.576 & 23.284 & 19.641 & 20.388 \\
\hline Walsh & 26.654 & 28.812 & 24.644 & 32.510 & $\mathbf{2 3 . 6 1 4}$ & 24.616 \\
\hline M Haar & 30.963 & 34.061 & $\mathbf{2 8 . 7 7 1}$ & 65.177 & 31.744 & 0.000 \\
\hline JPEG & 68.723 & 68.299 & $\mathbf{6 7 . 2 4 1}$ & 74.670 & 69.165 & 67.959 \\
\hline VQ compression & 44.388 & 44.104 & $\mathbf{4 1 . 0 9 4}$ & 58.687 & 43.924 & 42.036 \\
\hline DCT wavelet & 64.838 & 72.287 & 63.436 & 75.914 & 62.735 & 20.065 \\
\hline
\end{tabular}


Table 8. MAE between embedded and extracted watermark against compression using Real Fourier column transform and Real Fourier wavelet column transform

\begin{tabular}{|c|c|c|c|c|c|c|}
\hline \multirow[b]{2}{*}{ Compression using } & \multicolumn{5}{|c|}{ Real Fourier wavelet column transform } & \multirow{2}{*}{ Column Real Fourier Transform } \\
\hline & $(\mathbf{1 6 , 1 6})$ & $(32,8)$ & $(8,32)$ & $(64,4)$ & $(4,64)$ & \\
\hline DCT & 5.495 & 6.046 & 3.672 & 6.980 & 5.548 & 0.167 \\
\hline DST & 5.498 & 5.912 & 3.706 & 7.124 & 5.560 & 0.391 \\
\hline Walsh & 13.917 & 13.082 & 11.817 & 11.377 & 12.548 & 12.833 \\
\hline M Haar & 25.908 & 10.093 & 26.150 & 11.565 & 18.429 & 44.482 \\
\hline JPEG & 70.272 & 63.356 & 71.102 & $\mathbf{5 9 . 8 3 5}$ & 66.141 & 78.730 \\
\hline VQ compression & 48.977 & 51.569 & 55.715 & 50.642 & 51.726 & 62.944 \\
\hline DCT wavelet & 21.530 & 36.379 & 37.603 & 40.610 & 34.031 & 1.201 \\
\hline
\end{tabular}

Behaviour of orthogonal row transforms and their row wavelet transforms against compression attack in the

form MAE values are given below in Table 9 to Table 13.

Table 9. MAE between embedded and extracted watermark against compression, using DCT row transform and DCT wavelet row transform

\begin{tabular}{|c|c|c|c|c|c|c|}
\hline \multirow{2}{*}{ Compression using } & \multicolumn{5}{|c|}{ DCT wavelet } & \multirow{2}{*}{ Row DCT } \\
\cline { 2 - 6 } & $\mathbf{( 1 6 , 1 6 )}$ & $\mathbf{( 3 2 , 8 )}$ & $\mathbf{( 8 , 3 2})$ & $\mathbf{( 6 4 , 4 )}$ & $\mathbf{( 4 , 6 4 )}$ & \\
\hline DCT & 6.485 & 9.029 & 4.637 & 12.259 & $\mathbf{2 . 9 6 0}$ & 0.000 \\
\hline DST & 6.509 & 8.999 & 4.655 & 12.695 & $\mathbf{3 . 0 3 5}$ & 0.509 \\
\hline Walsh & 16.707 & 20.532 & 13.574 & 15.259 & $\mathbf{1 2 . 5 2 8}$ & 15.797 \\
\hline M Haar & 27.361 & 32.886 & 24.698 & $\mathbf{2 0 . 7 9 2}$ & 33.989 & 50.709 \\
\hline JPEG & 69.099 & 69.111 & 71.109 & $\mathbf{6 2 . 6 2 9}$ & 76.094 & 84.252 \\
\hline VQ compression & 59.801 & 49.102 & 61.160 & $\mathbf{4 0 . 1 5 0}$ & 62.164 & 72.610 \\
\hline DCT wavelet & $\mathbf{0 . 0 0 0}$ & 31.713 & 50.222 & 34.372 & 42.518 & 58.686 \\
\hline
\end{tabular}

Table 10. MAE between embedded and extracted watermark against compression using Haar row transform and Haar wavelet row transform

\begin{tabular}{|c|c|c|c|c|c|c|}
\hline \multirow{2}{*}{ Compression using } & \multicolumn{5}{|c|}{ Haar wavelet row transform } & \multirow{2}{*}{ Row Haar } \\
\cline { 2 - 6 } & $\mathbf{( 1 6 , 1 6 )}$ & $\mathbf{( 3 2 , 8 )}$ & $\mathbf{( 8 , 3 2})$ & $\mathbf{( 6 4 , 4 )}$ & $\mathbf{( 4 , 6 4 )}$ & \\
\hline DCT & 2.081 & 1.941 & 3.091 & $\mathbf{1 . 8 4 3}$ & 3.376 & 1.724 \\
\hline DST & 2.265 & 1.865 & 3.340 & $\mathbf{1 . 8 0 6}$ & 3.464 & 1.856 \\
\hline Walsh & 2.311 & $\mathbf{1 . 1 1 3}$ & 4.284 & 2.511 & 2.922 & 3.212 \\
\hline M Haar & 0.000 & 0.000 & 0.000 & 0.000 & 0.000 & 0.000 \\
\hline JPEG & $\mathbf{5 9 . 7 0 5}$ & 61.015 & 60.675 & 62.003 & 60.250 & 62.482 \\
\hline VQ compression & $\mathbf{3 9 . 2 6 5}$ & 43.542 & 41.548 & 40.710 & 39.806 & 43.824 \\
\hline DCT wavelet & $\mathbf{4 1 . 5 1 0}$ & 42.423 & 46.681 & 43.776 & 47.210 & 45.078 \\
\hline
\end{tabular}

Table 11. MAE between embedded and extracted watermark against compression using Walsh row transform and Walsh wavelet row transform

\begin{tabular}{|c|c|c|c|c|c|c|}
\hline \multirow{2}{*}{ Compression using } & \multicolumn{5}{|c|}{ Walsh wavelet row transform } & \multirow{2}{*}{ Row Walsh Transform } \\
\cline { 2 - 6 } & $\mathbf{( 1 6 , 1 6 )}$ & $\mathbf{( 3 2 , 8 )}$ & $\mathbf{( 8 , 3 2})$ & $\mathbf{( 6 4 , 4 )}$ & $\mathbf{( 4 , 6 4 )}$ & \\
\hline DCT & 1.282 & 1.591 & 1.342 & $\mathbf{1 . 2 1 5}$ & $\mathbf{1 . 2 1 5}$ & 1.257 \\
\hline DST & 1.393 & 1.714 & 1.417 & $\mathbf{1 . 2 7 0}$ & $\mathbf{1 . 2 7 0}$ & 1.324 \\
\hline Walsh & $\mathbf{0 . 0 0 0}$ & $\mathbf{0 . 0 0 0}$ & $\mathbf{0 . 0 0 0}$ & $\mathbf{0 . 0 0 0}$ & $\mathbf{0 . 0 0 0}$ & 0.000 \\
\hline M Haar & $\mathbf{0 . 0 0 0}$ & $\mathbf{0 . 0 0 0}$ & $\mathbf{0 . 0 0 0}$ & $\mathbf{0 . 0 0 0}$ & $\mathbf{0 . 0 0 0}$ & 8.651 \\
\hline JPEG & 65.212 & $\mathbf{6 4 . 9 3 1}$ & 65.047 & 67.027 & 67.027 & 69.352 \\
\hline VQ compression & $\mathbf{4 6 . 6 0 1}$ & 47.172 & 57.147 & 48.981 & 48.980 & 60.759 \\
\hline DCT wavelet & 58.872 & $\mathbf{5 4 . 5 8 9}$ & 54.823 & 58.150 & 58.15004 & 56.238 \\
\hline
\end{tabular}

Table 12. MAE between embedded and extracted watermark against compression using Kekre row transform and Kekre wavelet row transform

\begin{tabular}{|c|c|c|c|c|c|c|}
\hline \multirow{2}{*}{ Compression using } & \multicolumn{5}{|c|}{ Kekre wavelet row transform } & \multirow{2}{*}{ Row Kekre Transform } \\
\cline { 2 - 6 } & $\mathbf{( 1 6 , 1 6 )}$ & $\mathbf{( 3 2 , 8 )}$ & $\mathbf{( 8 , 3 2 )}$ & $\mathbf{( 6 4 , 4 )}$ & $\mathbf{( 4 , 6 4 )}$ & 19.405 \\
\hline DCT & $\mathbf{1 9 . 2 5 7}$ & 19.532 & 19.276 & 25.992 & 20.752 & 19.302 \\
\hline DST & $\mathbf{1 9 . 1 9 7}$ & 19.332 & 19.466 & 26.053 & 20.781 & 23.658 \\
\hline Walsh & 25.433 & 27.359 & 26.464 & 37.167 & $\mathbf{2 5 . 2 9 4}$ & 0.000 \\
\hline M Haar & 28.777 & 42.377 & 26.758 & 61.405 & 31.084 & 70.114 \\
\hline JPEG & 70.477 & 70.738 & $\mathbf{6 7 . 9 3 0}$ & 71.516 & 70.081 & 50.957 \\
\hline VQ compression & 44.323 & 46.939 & $\mathbf{4 1 . 9 2 1}$ & 47.051 & 46.289 & 64.260 \\
\hline DCT wavelet & 64.917 & 67.332 & $\mathbf{6 2 . 2 5 4}$ & 76.033 & 65.282 & \\
\hline
\end{tabular}


Table 13. MAE between embedded and extracted watermark against compression using Real Fourier row transform and Real Fourier wavelet row transform

\begin{tabular}{|c|c|c|c|c|c|c|}
\hline \multirow{2}{*}{ Compression using } & \multicolumn{9}{|c|}{ Real Fourier wavelet row transform } & \multirow{2}{*}{ Row Real Fourier Transform } \\
\cline { 2 - 6 } & $\mathbf{( 1 6 , 1 6 )}$ & $\mathbf{( 3 2 , 8 )}$ & $\mathbf{( 8 , 3 2 )}$ & $\mathbf{( 6 4 , 4 )}$ & $\mathbf{( 4 , 6 4 )}$ & \\
\hline DCT & 5.247 & 4.623 & 3.358 & 6.193 & $\mathbf{2 . 4 0 7}$ & 0.151 \\
\hline DST & 5.272 & 4.633 & 3.413 & 6.452 & $\mathbf{2 . 4 3 5}$ & 0.415 \\
\hline Walsh & 13.259 & 11.291 & 11.610 & $\mathbf{9 . 9 7 8}$ & 13.155 & 14.995 \\
\hline M Haar & 18.767 & 10.453 & 18.915 & $\mathbf{1 2 . 6 5 0}$ & 24.693 & 43.129 \\
\hline JPEG & 69.039 & 66.405 & 70.126 & $\mathbf{5 8 . 7 5 8}$ & 72.301 & 83.379 \\
\hline VQ compression & 56.274 & 46.621 & 58.937 & $\mathbf{3 9 . 1 8 0}$ & 60.879 & 63.491 \\
\hline DCT wavelet & $\mathbf{2 2 . 3 6 0}$ & 35.989 & 38.491 & 30.266 & 53.169 & 62.269 \\
\hline
\end{tabular}

\section{B. Cropping of Watermarked Images}

Cropping of watermarked images is performed in two ways. First is cropping at corners of image wherein $16 \times 16$ size portion is cropped at each corner of an image. To observe the effect of increased amount of cropping, $32 \times 32$ size squares are also cropped at four corners of an image. Second way is cropping $32 \times 32$ size portion from an image at its center. Extracted watermark from such cropped watermarked images are compared with embedded watermark by calculating MAE between the two. Result images of column DCT and column DCT wavelet and row DCT and Row DCT wavelet against cropping 16x16 portions at corners are shown in Fig. 4. For each recovered watermark, MAE and wavelet transforms (column and row), the combination of component transform which gives minimum MAE is mentioned below the image.

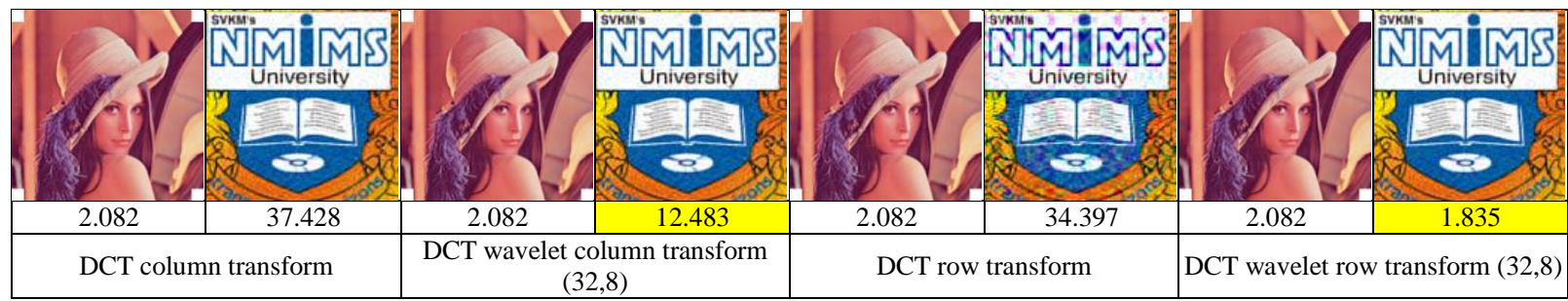

Fig. 4. Result images for cropping 16x16 portions at corners when DCT column, DCT wavelet column, DCT row and DCT wavelet row transform is used to embed watermark

From Fig. 4, improvement in robustness against cropping attack using wavelet transform is clearly noticeable from MAE values. In case of column DCT wavelet and row DCT wavelet transform $(32,8)$ combination of component DCT matrices gives highest robustness.
Table 14 shows MAE values for extracted watermark from different types of cropping attack when column DCT and column DCT wavelet generated from different combinations of DCT is used for embedding and extraction process.

Table 14. MAE between embedded and extracted watermark against cropping attack using column DCT and column DCT wavelet transform

\begin{tabular}{|c|c|c|c|c|c|c|}
\hline \multirow{2}{*}{ Cropping type } & \multicolumn{5}{|c|}{ Column DCT wavelet } & \multirow{2}{*}{ Column DCT } \\
\cline { 2 - 6 } & $\mathbf{( 1 6 , 1 6 )}$ & $\mathbf{( 3 2 , 8 )}$ & $\mathbf{( 8 , 3 2})$ & $\mathbf{( 6 4 , 4 )}$ & $\mathbf{( 4 , 6 4 )}$ & \\
\hline $16 \times 16$ crop & 14.470 & $\mathbf{1 2 . 4 8 3}$ & 13.657 & 15.214 & 32.891 & 37.428 \\
\hline $32 \times 32$ crop & 37.386 & 25.532 & $\mathbf{7 . 5 8 1}$ & 31.046 & 56.120 & 69.534 \\
\hline $32 \times 32$ crop center & 24.271 & 24.572 & 24.166 & $\mathbf{0 . 0 0 0}$ & 35.549 & 62.228 \\
\hline
\end{tabular}

From Table 14, it is observed that column DCT wavelet transform performs far better than column DCT especially for $32 \times 32$ cropping done at the center of an image.

Table 15. MAE between embedded and extracted watermark against cropping attack using row DCT and row DCT wavelet transform.

\begin{tabular}{|c|c|c|c|c|c|c|}
\hline \multirow{2}{*}{ Cropping type } & \multicolumn{5}{|c|}{ Row DCT wavelet } & \multirow{2}{*}{ Row DCT } \\
\cline { 2 - 6 } & $\mathbf{( 1 6 , 1 6 )}$ & $\mathbf{( 3 2 , 8 )}$ & $\mathbf{( 8 , 3 2 )}$ & $\mathbf{( 6 4 , 4 )}$ & $(\mathbf{4 , 6 4})$ & \\
\hline $16 \times 16$ crop & 2.692 & $\mathbf{1 . 8 3 5}$ & 25.821 & 3.059 & 33.239 & 34.397 \\
\hline $32 \times 32$ crop & 25.900 & 16.429 & $\mathbf{7 . 0 1 1}$ & 19.618 & 63.099 & 75.321 \\
\hline $32 \times 32$ crop center & 3.441 & 3.310 & 21.118 & $\mathbf{0 . 0 0 0}$ & 45.252 & 56.789 \\
\hline
\end{tabular}

Table 16 to Table 19 show the performance of other column wavelets and corresponding orthogonal column
Similar observations can be made for row DCT wavelet transform and row DCT against cropping attack from Table 15. transforms against cropping attack. For different types of cropping performed on watermarked images, different 
combinations used for wavelet generation are observed to be giving better MAE values. In all cases, column wavelet transform performs exceptionally well over simple column transform.

Table 16. MAE between embedded and extracted watermark against cropping attack using column Haar and column Haar wavelet transform.

\begin{tabular}{|c|c|c|c|c|c|c|}
\hline \multirow{2}{*}{ Cropping type } & \multicolumn{5}{|c|}{ Column Haar wavelet } & \multirow{2}{*}{ Column Haar } \\
\cline { 2 - 6 } & $\mathbf{( 1 6 , 1 6 )}$ & $\mathbf{( 3 2 , 8 )}$ & $\mathbf{( 8 , 3 2 )}$ & $\mathbf{( 6 4 , 4 )}$ & $\mathbf{( 4 , 6 4 )}$ & \\
\hline 16x16 crop & $\mathbf{0 . 2 9 3}$ & 2.099 & 4.459 & 17.323 & 1.529 & 18.060 \\
\hline $32 \times 32$ crop & 19.375 & 6.737 & 12.159 & 24.187 & $\mathbf{5 . 6 0 6}$ & 25.467 \\
\hline $32 \times 32$ crop center & 0.244 & 1.636 & 5.816 & $\mathbf{0 . 0 0 0}$ & 3.557 & 0.000 \\
\hline
\end{tabular}

Table 17. MAE between embedded and extracted watermark against cropping attack using column Walsh and column Walsh wavelet transform

\begin{tabular}{|c|c|c|c|c|c|c|}
\hline \multirow{2}{*}{ Cropping type } & \multicolumn{5}{|c|}{ Column Walsh wavelet } & \multirow{2}{*}{ Column Walsh } \\
\cline { 2 - 6 } & $\mathbf{( 1 6 , 1 6 )}$ & $\mathbf{( 3 2 , 8 )}$ & $\mathbf{( 8 , 3 2 )}$ & $\mathbf{( 6 4 , 4 )}$ & $\mathbf{( 4 , 6 4 )}$ & \\
\hline $16 \times 16$ crop & 10.177 & 14.119 & $\mathbf{5 . 4 1 8}$ & 9.905 & 9.905 & 21.832 \\
\hline $32 \times 32$ crop & 26.448 & 25.957 & $\mathbf{6 . 8 9 3}$ & 19.766 & 19.766 & 38.805 \\
\hline $32 \times 32$ crop center & 12.256 & $\mathbf{6 . 1 2 8}$ & 6.534 & 8.306 & 8.306 & 26.087 \\
\hline
\end{tabular}

Table 18. MAE between embedded and extracted watermark against cropping attack using column Kekre transform and column Kekre wavelet transform

\begin{tabular}{|c|c|c|c|c|c|c|}
\hline \multirow{2}{*}{ Cropping type } & \multicolumn{4}{|c|}{ Column Kekre wavelet } & \multirow{2}{*}{ Column Kekre Transform } \\
\cline { 2 - 6 } & $\mathbf{( 1 6 , 1 6 )}$ & $\mathbf{( 3 2 , 8 )}$ & $\mathbf{( 8 , 3 2 )}$ & $\mathbf{( 6 4 , 4 )}$ & $\mathbf{( 4 , 6 4 )}$ & \\
\hline $16 \times 16$ crop & 3.955 & $\mathbf{3 . 4 9 4}$ & 29.290 & 16.105 & 12.289 & 19.484 \\
\hline $32 \times 32$ crop & 39.768 & 6.803 & $\mathbf{2 . 1 1 5}$ & 25.121 & 199.689 & 63.456 \\
\hline $32 \times 32$ crop center & 3.316 & 13.725 & 97.620 & $\mathbf{0 . 0 0 0}$ & 25.098 & 126.096 \\
\hline
\end{tabular}

Table 19. MAE between embedded and extracted watermark against cropping attack using column RFT and column RFT wavelet transform

\begin{tabular}{|c|c|c|c|c|c|c|}
\hline \multirow{2}{*}{ Cropping type } & \multicolumn{5}{|c|}{ Column RFT wavelet } & \multirow{2}{*}{ Column RFT } \\
\cline { 2 - 6 } & $\mathbf{( 1 6 , 1 6 )}$ & $\mathbf{( 3 2 , 8 )}$ & $\mathbf{( 8 , 3 2 )}$ & $\mathbf{( 6 4 , 4 )}$ & $\mathbf{( 4 , 6 4 )}$ & \\
\hline $16 x 16$ crop & 11.874 & $\mathbf{1 2 . 6 6 0}$ & 15.532 & 14.692 & 13.689 & 31.881 \\
\hline $32 \times 32$ crop & 34.827 & 15.781 & $\mathbf{5 . 3 8 8}$ & 39.109 & 23.776 & 67.620 \\
\hline $32 \times 32$ crop center & 24.936 & 11.443 & 11.921 & $\mathbf{0 . 0 0 0}$ & 12.075 & 52.352 \\
\hline
\end{tabular}

Performance of row wavelet and respective orthogonal row transform are compared in Table 20 to Table 23. From Table 20 to Table 23, once again robustness of row wavelet transform is observed to be significantly better than simple row transform.

Table 20. MAE between embedded and extracted watermark against cropping attack using row Haar and row Haar wavelet transform

\begin{tabular}{|c|c|c|c|c|c|c|}
\hline \multirow{2}{*}{ Cropping type } & \multicolumn{5}{|c|}{ Row Haar wavelet } & \multirow{2}{*}{ Row Haar } \\
\cline { 2 - 6 } & $\mathbf{( 1 6 , 1 6 )}$ & $\mathbf{( 3 2 , 8 )}$ & $\mathbf{( 8 , 3 2 )}$ & $\mathbf{( 6 4 , 4 )}$ & $\mathbf{( 4 , 6 4 )}$ & \\
\hline $16 \times 16$ crop & 5.037 & 4.499 & 5.230 & $\mathbf{4 . 0 4 9}$ & 4.270 & 4.305 \\
\hline $32 \times 32$ crop & 21.545 & $\mathbf{8 . 6 5 1}$ & 12.046 & 19.423 & 18.277 & 24.729 \\
\hline $32 \times 32$ crop center & 2.984 & 3.253 & 1.571 & $\mathbf{0 . 0 0 0}$ & 2.384 & 0.000 \\
\hline
\end{tabular}

Table 21. MAE between embedded and extracted watermark against cropping attack using row Walsh and row Walsh wavelet transform

\begin{tabular}{|c|c|c|c|c|c|c|}
\hline \multirow{2}{*}{ Cropping type } & \multicolumn{5}{|c|}{ Row Walsh wavelet } & \multirow{2}{*}{ Row Walsh } \\
\cline { 2 - 6 } & $\mathbf{( 1 6 , 1 6 )}$ & $\mathbf{( 3 2 , 8 )}$ & $\mathbf{( 8 , 3 2 )}$ & $\mathbf{( 6 4 , 4 )}$ & $\mathbf{( 4 , 6 4 )}$ & \\
\hline $16 \times 16$ crop & $\mathbf{4 . 0 4 9}$ & 5.275 & 7.439 & 14.327 & 14.326 & 19.976 \\
\hline $32 \times 32$ crop & $\mathbf{1 3 . 5 1 8}$ & 31.544 & 15.550 & 35.988 & 35.987 & 37.892 \\
\hline $32 \times 32$ crop center & 4.973 & $\mathbf{2 . 5 4 9}$ & 26.627 & 21.477 & 21.477 & 31.042 \\
\hline
\end{tabular}

Table 22. MAE between embedded and extracted watermark against cropping attack using row Kekre transform and row Kekre wavelet transform

\begin{tabular}{|c|c|c|c|c|c|c|}
\hline \multirow{2}{*}{ Cropping type } & \multicolumn{5}{|c|}{ Row Kekre wavelet } & \multirow{2}{*}{ Row Kekre Transform } \\
\cline { 2 - 6 } & $\mathbf{( 1 6 , 1 6 )}$ & $\mathbf{( 3 2 , 8 )}$ & $\mathbf{( 8 , 3 2 )}$ & $\mathbf{( 6 4 , 4 )}$ & $\mathbf{( 4 , 6 4 )}$ & \\
\hline $16 \times 16$ crop & 14.207 & 1.892 & 20.647 & $\mathbf{4 . 8 3 5}$ & 50.308 & 40.301 \\
\hline $32 \times 32$ crop & 18.353 & 6.368 & $\mathbf{5 . 5 3 1}$ & 10.123 & 202.193 & 112.438 \\
\hline $32 \times 32$ crop center & 14.940 & 14.125 & 129.946 & $\mathbf{0 . 0 0 0}$ & 31.970 & 129.552 \\
\hline
\end{tabular}

Table 23. MAE between embedded and extracted watermark against cropping attack using row RFT and row RFT wavelet transform

\begin{tabular}{|c|c|c|c|c|c|c|}
\hline \multirow{2}{*}{ Cropping type } & \multicolumn{5}{|c|}{ Row RFT wavelet } & \multirow{2}{*}{ Row RFT } \\
\cline { 2 - 6 } & $\mathbf{( 1 6 , 1 6 )}$ & $\mathbf{( 3 2 , 8 )}$ & $\mathbf{( 8 , 3 2 )}$ & $\mathbf{( 6 4 , 4 )}$ & $\mathbf{( 4 , 6 4 )}$ & \\
\hline 16x16 crop & 4.050 & $\mathbf{2 . 7 7 4}$ & 13.907 & 7.404 & 45.755 & 42.124 \\
\hline $32 \times 32$ crop & $\mathbf{1 8 . 3 8 5}$ & 24.856 & 25.190 & 20.668 & 74.147 & 68.542 \\
\hline 32 x32 crop center & 2.028 & 3.820 & 29.133 & $\mathbf{0 . 0 0 0}$ & 46.174 & 56.045 \\
\hline
\end{tabular}




\section{Noise addition to watermarked images}

Two types of noises are added to watermarked images namely binary distributed random noise having magnitude 1or -1 . Binary distributed random noise is added to watermarked images with different run lengths like run length 1 to 10,5 to 50 and 10 to 100 to study the impact of increased noise. Another type of noise added to watermarked images is Gaussian distributed run length noise. It has discrete magnitude in the range $[-2,2]$. Watermarked images and extracted watermark from binary distributed run length noise with run length 10 to 100 are shown in Fig. 5 using column DCT and column DCT wavelet used for embedding watermark.

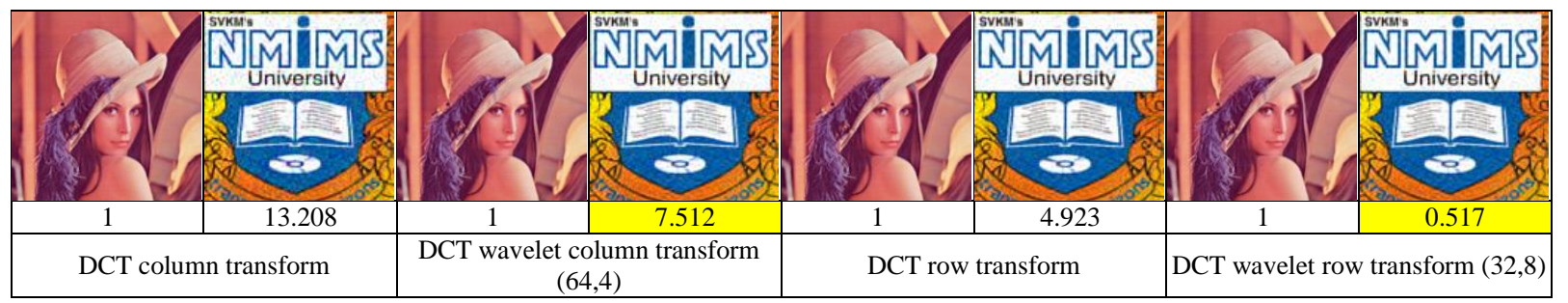

Fig. 5. Result images for binary distributed run length noise with run length 10 to 100 attack when DCT column, DCT wavelet column, DCT row and DCT wavelet row transform is used to embed watermark

From Fig. 5, it can be seen that use of column and row DCT wavelet transform significantly improves the robustness over use of column and row DCT used for embedding watermark. Table 24 shows MAE between embedded and extracted watermark after adding different type of noises to watermarked image for column DCT and column DCT wavelet transform.

Table 24. MAE values between embedded and extracted watermark from different types of binary run length noises and Gaussian distributed noise added to watermarked images using column DCT and column DCT wavelet for embedding and extraction

\begin{tabular}{|c|c|c|c|c|c|c|}
\hline \multirow{2}{*}{ Noise type } & \multicolumn{5}{|c|}{ Column DCT wavelet } & \multirow{2}{*}{ Column DCT } \\
\cline { 2 - 6 } & $\mathbf{( 1 6 , 1 6 )}$ & $\mathbf{( 3 2 , 8 )}$ & $\mathbf{( 8 , 3 2 )}$ & $\mathbf{( 6 4 , 4 )}$ & $\mathbf{( 4 , 6 4 )}$ & \\
\hline BRLN & $\mathbf{0 . 0 0 0}$ & $\mathbf{0 . 0 0 0}$ & $\mathbf{0 . 0 0 0}$ & $\mathbf{0 . 0 0 0}$ & $\mathbf{0 . 0 0 0}$ & 0.000 \\
\hline BRLN (5to50) & 8.277 & $\mathbf{7 . 1 0 8}$ & 8.366 & 7.662 & 10.306 & 13.784 \\
\hline BRLN (10 to 100) & 8.191 & 7.564 & 9.443 & $\mathbf{7 . 5 1 2}$ & 9.914 & 13.208 \\
\hline GRLN & 0.728 & $\mathbf{0 . 5 3 4}$ & 0.908 & 0.560 & 0.941 & 1.286 \\
\hline
\end{tabular}

From Table 24 it can be observed that wavelet transform works equally well for smaller run length and significantly better than orthogonal transform for increased run length noise. For Gaussian distributed run length noise also all combinations tried for wavelet transform using DCT give better robustness.
Table 25 shows performance of row DCT and row DCT wavelet transform against noise addition attack in the form of MAE between embedded and extracted watermark. From Table 25 it can be seen that wavelet transform in row version also performs better than row DCT transform.

Table 25. MAE values between embedded and extracted watermark from noise added watermarked images when Row DCT and Row DCT wavelet transform is used for embedding and extracting watermark

\begin{tabular}{|c|c|c|c|c|c|c|}
\hline \multirow{2}{*}{ Noise type } & \multicolumn{5}{|c|}{ Row DCT wavelet } & \multirow{2}{*}{ Row DCT } \\
\cline { 2 - 6 } & $\mathbf{( 1 6 , 1 6 )}$ & $\mathbf{( 3 2 , 8 )}$ & $\mathbf{( 8 , 3 2 )}$ & $\mathbf{( 6 4 , 4 )}$ & $\mathbf{( 4 , 6 4 )}$ & \\
\hline BRLN & $\mathbf{3 . 9 1 6}$ & 4.378 & 4.614 & 4.245 & 4.575 & 6.573 \\
\hline BRLN (5to50) & 1.963 & 1.695 & 2.122 & $\mathbf{1 . 6 6 7}$ & 2.525 & 2.539 \\
\hline BRLN (10 to 100) & 1.195 & $\mathbf{1 . 0 5 9}$ & 1.344 & 1.071 & 1.797 & 2.071 \\
\hline GRLN & 7.163 & 6.036 & 8.123 & $\mathbf{5 . 0 8 9}$ & 8.930 & 11.431 \\
\hline
\end{tabular}

Table 26 to Table 29 show MAE values between embedded and recovered watermark when column Haar and its wavelet, column Walsh and its wavelet, column Kekre transform and its wavelet and column RFT and its wavelet are used to embed and extract watermark and different types of noise addition is done to watermarked images. From these tables, it is concluded that wavelet transforms obtained from orthogonal transforms are more robust to noise addition attacks than corresponding orthogonal transforms. Different combinations of orthogonal transforms used in generation of wavelet give different MAE values. But most of them are smaller than orthogonal column transforms or negligibly higher. 
Table 26. MAE values between embedded and extracted watermark from different types of noises added to watermarked images using column Haar and column Haar wavelet for embedding and extraction

\begin{tabular}{|c|c|c|c|c|c|c|}
\hline \multirow{2}{*}{ Noise type } & \multicolumn{5}{|c|}{ Column Haar wavelet } & \multirow{2}{*}{ Column Haar } \\
\cline { 2 - 6 } & $\mathbf{( 1 6 , 1 6 )}$ & $\mathbf{( 3 2 , 8 )}$ & $\mathbf{( 8 , 3 2 )}$ & $\mathbf{( 6 4 , 4 )}$ & $\mathbf{( 4 , 6 4 )}$ & \\
\hline BRLN & 0.000 & 0.000 & 0.000 & 0.000 & 0.000 & 0.000 \\
\hline BRLN (5to50) & 5.366 & 5.378 & $\mathbf{5 . 1 9 1}$ & 8.340 & 5.763 & 6.947 \\
\hline BRLN (10 to 100) & $\mathbf{5 . 0 4 5}$ & 5.634 & 5.768 & 7.279 & 5.673 & 7.496 \\
\hline GRLN & 0.493 & 0.370 & 0.260 & $\mathbf{0 . 2 1 7}$ & 0.388 & 0.201 \\
\hline
\end{tabular}

Table 27. MAE values between embedded and extracted watermark from different types of noises added to watermarked images using column Walsh and column Walsh wavelet for embedding and extraction

\begin{tabular}{|c|c|c|c|c|c|c|}
\hline \multirow{2}{*}{ Noise type } & \multicolumn{5}{|c|}{ Column Walsh wavelet } & \multirow{2}{*}{ Column Walsh } \\
\cline { 2 - 6 } & $\mathbf{( 1 6 , 1 6 )}$ & $\mathbf{( 3 2 , 8 )}$ & $\mathbf{( 8 , 3 2 )}$ & $\mathbf{( 6 4 , 4 )}$ & $\mathbf{( 4 , 6 4 )}$ & \\
\hline BRLN & 0.000 & 0.000 & 0.000 & 0.000 & 0.000 & 0.000 \\
\hline BRLN (5to50) & $\mathbf{6 . 4 8 1}$ & 6.714 & 7.757 & 6.984 & 6.984 & 11.445 \\
\hline BRLN (10 to 100) & $\mathbf{6 . 5 3 7}$ & 7.017 & 7.231 & 6.928 & 6.928 & 10.942 \\
\hline GRLN & 0.915 & $\mathbf{0 . 5 5 8}$ & 1.116 & 0.863 & 0.863 & 2.176 \\
\hline
\end{tabular}

Table 28. MAE values between embedded and extracted watermark from different types of noises added to watermarked images using column Kekre Transform and column Kekre wavelet for embedding and extraction

\begin{tabular}{|c|c|c|c|c|c|c|}
\hline \multirow{2}{*}{ Noise type } & \multicolumn{5}{|c|}{ Column Kekre wavelet } & \multirow{2}{*}{ Column Kekre Transform } \\
\cline { 2 - 6 } & $\mathbf{( 1 6 , 1 6 )}$ & $\mathbf{( 3 2 , 8 )}$ & $\mathbf{( 8 , 3 2 )}$ & $\mathbf{( 6 4 , 4 )}$ & $\mathbf{( 4 , 6 4 )}$ & \\
\hline BRLN & $\mathbf{0 . 0 0 0}$ & $\mathbf{0 . 0 0 0}$ & $\mathbf{0 . 0 0 0}$ & $\mathbf{0 . 0 0 0}$ & $\mathbf{0 . 0 0 0}$ & 0.000 \\
\hline BRLN (5to50) & 5.163 & 5.520 & $\mathbf{4 . 9 4 8}$ & 7.749 & 5.117 & 5.475 \\
\hline BRLN (10 to 100) & 5.369 & 5.628 & $\mathbf{4 . 8 1 5}$ & 8.304 & 5.235 & 5.476 \\
\hline GRLN & 0.720 & 0.287 & 1.353 & $\mathbf{0 . 0 8 2}$ & 1.914 & 3.787 \\
\hline
\end{tabular}

Table 29. MAE values between embedded and extracted watermark from different types of noises added to watermarked images using column RFT and column RFT wavelet for embedding and extraction

\begin{tabular}{|c|c|c|c|c|c|c|}
\hline \multirow{2}{*}{ Noise type } & \multicolumn{5}{|c|}{ Column RFT wavelet } & \multirow{2}{*}{ Column RFT } \\
\cline { 2 - 6 } & $\mathbf{( 1 6 , 1 6 )}$ & $\mathbf{( 3 2 , 8 )}$ & $\mathbf{( 8 , 3 2 )}$ & $\mathbf{( 6 4 , 4 )}$ & $\mathbf{( 4 , 6 4 )}$ & \\
\hline BRLN & $\mathbf{0 . 0 0 0}$ & $\mathbf{0 . 0 0 0}$ & $\mathbf{0 . 0 0 0}$ & $\mathbf{0 . 0 0 0}$ & $\mathbf{0 . 0 0 0}$ & 0.000 \\
\hline BRLN (5to50) & 8.128 & 7.324 & 9.278 & $\mathbf{6 . 9 3 9}$ & 7.917 & 14.901 \\
\hline BRLN (10 to 100) & 7.789 & $\mathbf{6 . 6 3 2}$ & 9.200 & 7.831 & 7.863 & 12.788 \\
\hline GRLN & 0.547 & 0.753 & 1.087 & $\mathbf{0 . 3 7 1}$ & 0.689 & 1.453 \\
\hline
\end{tabular}

Similar response is observed by row wavelet show this response in the form MAE values. transforms over row transforms. Table 30 to Table 33

Table 30. MAE values between embedded and extracted watermark from noise added watermarked images when Row Haar and Row Haar wavelet transform is used for embedding and extracting watermark

\begin{tabular}{|c|c|c|c|c|c|c|}
\hline \multirow{2}{*}{ Noise type } & \multicolumn{5}{|c|}{ Row Haar wavelet } & \multirow{2}{*}{ Row Haar } \\
\cline { 2 - 6 } & $\mathbf{( 1 6 , 1 6 )}$ & $\mathbf{( 3 2 , 8 )}$ & $\mathbf{( 8 , 3 2 )}$ & $\mathbf{( 6 4 , 4 )}$ & $\mathbf{( 4 , 6 4 )}$ & \\
\hline BRLN & 4.399 & 3.925 & $\mathbf{3 . 4 2 7}$ & 3.881 & 3.465 & 4.642 \\
\hline BRLN (5to50) & 1.145 & $\mathbf{1 . 0 8 8}$ & 1.526 & 1.825 & 2.018 & 1.843 \\
\hline BRLN (10 to 100) & 0.981 & 0.735 & 1.078 & 1.273 & $\mathbf{0 . 5 9 4}$ & 1.357 \\
\hline GRLN & $\mathbf{4 . 8 7 9}$ & 5.797 & 5.189 & 5.139 & 5.103 & 5.262 \\
\hline
\end{tabular}

Table 31. MAE values between embedded and extracted watermark from noise added watermarked images when Row Walsh and Row Walsh wavelet transform is used for embedding and extracting watermark

\begin{tabular}{|c|c|c|c|c|c|c|}
\hline \multirow{2}{*}{ Noise type } & \multicolumn{5}{|c|}{ Row Walsh wavelet } & \multirow{2}{*}{ Row Walsh } \\
\cline { 2 - 6 } & $\mathbf{( 1 6 , 1 6 )}$ & $\mathbf{( 3 2 , 8 )}$ & $\mathbf{( 8 , 3 2 )}$ & $\mathbf{( 6 4 , 4 )}$ & $\mathbf{( 4 , 6 4 )}$ & \\
\hline BRLN & 4.568 & $\mathbf{4 . 4 3 5}$ & 5.313 & 6.373 & 6.373 & 6.822 \\
\hline BRLN (5to50) & 2.036 & $\mathbf{1 . 8 2 5}$ & 2.554 & 2.799 & 2.799 & 3.549 \\
\hline BRLN (10 to 100) & 1.675 & $\mathbf{1 . 3 7 0}$ & 1.987 & 2.254 & 2.254 & 2.697 \\
\hline GRLN & 6.913 & $\mathbf{6 . 2 6 8}$ & 7.289 & 7.672 & 7.672 & 8.887 \\
\hline
\end{tabular}

Table 32. MAE values between embedded and extracted watermark from noise added watermarked images when Row Kekre transform and Row Kekre wavelet transform is used for embedding and extracting watermark

\begin{tabular}{|c|c|c|c|c|c|c|}
\hline \multirow{2}{*}{ Noise type } & \multicolumn{5}{|c|}{ Row Kekre wavelet } & \multirow{2}{*}{ Row Kekre Transform } \\
\cline { 2 - 6 } & $\mathbf{( 1 6 , 1 6 )}$ & $\mathbf{( 3 2 , 8 )}$ & $\mathbf{( 8 , 3 2 )}$ & $\mathbf{( 6 4 , 4 )}$ & $\mathbf{( 4 , 6 4 )}$ & \\
\hline BRLN & $\mathbf{4 . 7 4 4}$ & 5.342 & 5.301 & 5.075 & 5.379 & 6.244 \\
\hline BRLN (5to50) & 2.107 & 1.971 & 3.129 & $\mathbf{1 . 8 1 0}$ & 4.870 & 5.975 \\
\hline BRLN (10 to 100) & 1.886 & $\mathbf{0 . 8 3 4}$ & 2.255 & 1.186 & 3.516 & 5.802 \\
\hline GRLN & $\mathbf{5 . 2 0 6}$ & 5.912 & 5.494 & 7.036 & 5.576 & 5.601 \\
\hline
\end{tabular}


Table 33. MAE values between embedded and extracted watermark from noise added watermarked images when Row RFT and Row RFT wavelet transform is used for embedding and extracting watermark

\begin{tabular}{|c|c|c|c|c|c|c|}
\hline \multirow{2}{*}{ Noise type } & \multicolumn{5}{|c|}{ Row RFT wavelet } & \multirow{2}{*}{ Row RFT } \\
\cline { 2 - 6 } & $\mathbf{( 1 6 , 1 6 )}$ & $\mathbf{( 3 2 , 8 )}$ & $\mathbf{( 8 , 3 2 )}$ & $\mathbf{( 6 4 , 4 )}$ & $\mathbf{( 4 , 6 4 )}$ & \\
\hline BRLN & 4.385 & 4.032 & 5.119 & $\mathbf{3 . 3 9 5}$ & 5.293 & 5.966 \\
\hline BRLN (5to50) & 1.857 & 1.814 & 2.631 & $\mathbf{1 . 4 6 6}$ & 2.422 & 2.686 \\
\hline BRLN (10 to 100) & 1.701 & 1.024 & 1.762 & $\mathbf{0 . 3 3 7}$ & 1.903 & 2.057 \\
\hline GRLN & 7.036 & 6.111 & 7.426 & $\mathbf{5 . 0 4 1}$ & 9.018 & 10.453 \\
\hline
\end{tabular}

\section{Resizing attack on watermarked images}

Resizing attack is performed using three different techniques. First using bicubic interpolation in which watermarked image is doubled in size and then reduced back to its original size. Also watermarked image is zoomed to make it four times larger and then reduced back to its original size. Second approach used for resizing attack is using grid based image zooming technique proposed in [12]. In this approach image is doubled and reduced back to its original size. Third approach is using transform based image zooming technique [14]. In this third approach, watermarked image is doubled in size using different orthogonal transforms like DCT, DST, DFT, Hartley and Real Fourier Transform.

Fig. 6 shows the result images for grid based resizing attack when column and row versions of DCT and DCT wavelet are used for embedding and extracting watermark.

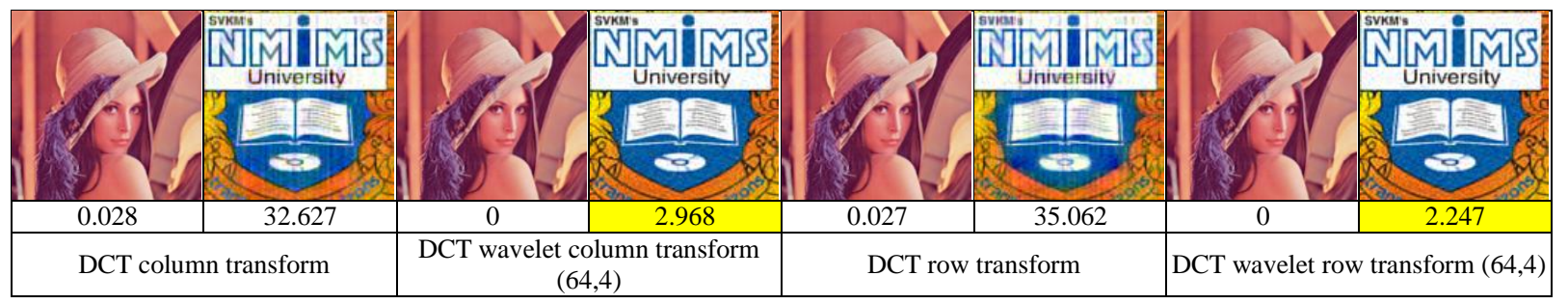

Fig. 6. Extracted watermark from grid based resized watermarked images when DCT column, DCT wavelet column, DCT row and DCT wavelet row transform is used to embed watermark

From Fig. 6, the difference between performances against grid based resizing attack when column/row DCT and column/row DCT wavelet is used for embedding watermark is clearly seen. DCT Wavelet transforms in column and row version provides more robustness than DCT column and row transform.
Table 34 and Table 35 show the MAE values between embedded and extracted watermark against various resizing attacks using column version and row version of DCT/ DCT wavelet transform respectively.

Table 34. MAE values against resizing attacks when column DCT and column DCT wavelet are used to embed and extract watermark

\begin{tabular}{|c|c|c|c|c|c|c|}
\hline \multirow{2}{*}{ Resizing type } & \multicolumn{5}{|c|}{ Column DCT wavelet } & \multirow{2}{*}{ Column DCT } \\
\cline { 2 - 6 } & $\mathbf{( 1 6 , 1 6 )}$ & $\mathbf{( 3 2 , 8 )}$ & $\mathbf{( 8 , 3 2 )}$ & $\mathbf{( 6 4 , 4 )}$ & $\mathbf{( 4 , 6 4 )}$ & \\
\hline Resize4 & $\mathbf{2 6 . 2 8 7}$ & 28.717 & 27.400 & 31.221 & 28.090 & 29.670 \\
\hline Resize2 & $\mathbf{2 7 . 0 2 8}$ & 29.494 & 28.178 & 32.038 & 28.875 & 30.502 \\
\hline DFT_resize2 & 1.566 & 1.798 & $\mathbf{1 . 5 3 6}$ & 1.755 & 1.793 & 2.023 \\
\hline grid resize2 & 4.398 & 3.365 & 5.305 & $\mathbf{2 . 9 6 8}$ & 8.566 & 32.627 \\
\hline
\end{tabular}

Table 35. MAE values against resizing attacks when row DCT and row DCT wavelet are used to embed and extract watermark

\begin{tabular}{|c|c|c|c|c|c|c|}
\hline \multirow{2}{*}{ Resizing type } & \multicolumn{5}{|c|}{ Row DCT wavelet } & \multirow{2}{*}{ Row DCT } \\
\cline { 2 - 6 } & $\mathbf{( 1 6 , 1 6 )}$ & $\mathbf{( 3 2 , 8 )}$ & $\mathbf{( 8 , 3 2 )}$ & $\mathbf{( 6 4 , 4 )}$ & $\mathbf{( 4 , 6 4 )}$ & \\
\hline Resize4 & 26.209 & 31.090 & $\mathbf{2 5 . 9 4 7}$ & 32.595 & 27.360 & 28.501 \\
\hline Resize2 & 26.899 & 31.924 & $\mathbf{2 6 . 6 6 1}$ & 33.464 & 28.127 & 29.294 \\
\hline DFT_resize2 & 1.903 & 1.693 & 1.791 & $\mathbf{1 . 4 4 5}$ & 2.252 & 2.515 \\
\hline grid resize2 & 4.253 & 3.358 & 5.527 & $\mathbf{2 . 2 4 7}$ & 9.375 & 35.062 \\
\hline
\end{tabular}

From Table 34 and Table 35, it is observed that column and row DCT wavelet transforms perform marginally better against resizing using bicubic interpolation attack and resizing using DFT. Against resizing using grid based interpolation, performance of column and row DCT wavelet is far better than column and row DCT respectively. Another noticeable observation regarding transform based resizing attack is that MAE between embed and extracted watermark is observed to be zero using DCT, DST, Hartley and Real Fourier Transform irrespective of the transform used for embedding and extraction process.

Table 36 to Table 39 show the performance of column wavelet transforms obtained from Haar, Walsh, Kekre 
transform and Real Fourier transform and corresponding column transforms respectively against different types of

resizing attack.

Table 36. MAE values against resizing attacks when column Haar and column Haar wavelet are used to embed and extract watermark

\begin{tabular}{|c|c|c|c|c|c|c|}
\hline \multirow{2}{*}{ Resizing type } & \multicolumn{5}{|c|}{ Column Haar wavelet } & \multirow{2}{*}{ Column Haar } \\
\cline { 2 - 6 } & $\mathbf{( 1 6 , 1 6 )}$ & $\mathbf{( 3 2 , 8 )}$ & $\mathbf{( 8 , 3 2 )}$ & $\mathbf{( 6 4 , 4 )}$ & $\mathbf{( 4 , 6 4 )}$ & \\
\hline Resize4 & $\mathbf{2 7 . 1 8 0}$ & 27.962 & 29.182 & 27.791 & 27.267 & 27.688 \\
\hline Resize2 & $\mathbf{2 7 . 9 1 2}$ & 28.705 & 29.962 & 28.532 & 27.994 & 28.422 \\
\hline DFT_resize2 & $\mathbf{1 . 0 8 6}$ & 1.129 & 1.128 & 1.230 & 1.091 & 1.199 \\
\hline grid resize2 & $\mathbf{3 . 0 2 9}$ & 3.048 & 3.087 & 4.319 & 3.179 & 4.339 \\
\hline
\end{tabular}

Table 37. MAE values against resizing attacks when column Walsh and column Walsh wavelet are used to embed and extract watermark

\begin{tabular}{|c|c|c|c|c|c|c|}
\hline \multirow{2}{*}{ Resizing type } & \multicolumn{5}{|c|}{ Column Walsh wavelet } & \multirow{2}{*}{ Column Walsh } \\
\cline { 2 - 7 } & $\mathbf{( 1 6 , 1 6 )}$ & $\mathbf{( 3 2 , 8 )}$ & $\mathbf{( 8 , 3 2 )}$ & $\mathbf{( 6 4 , 4 )}$ & $\mathbf{( 4 , 6 4 )}$ & \\
\hline Resize4 & 28.859 & 28.273 & 29.121 & 28.751 & 28.751 & 30.001 \\
\hline Resize2 & 29.675 & 29.049 & 29.934 & 29.552 & 29.552 & 30.836 \\
\hline DFT_resize2 & 1.206 & 1.203 & 1.043 & 1.151 & 1.151 & 1.625 \\
\hline grid resize2 & 8.105 & 6.222 & 11.381 & 8.570 & 8.570 & 55.839 \\
\hline
\end{tabular}

Table 38. MAE values against resizing attacks when column Kekre Transform and column Kekre wavelet Transform are used to embed and extract watermark

\begin{tabular}{|c|c|c|c|c|c|c|}
\hline \multirow{2}{*}{ Resizing type } & \multicolumn{5}{|c|}{ Column Kekre wavelet } & \multirow{2}{*}{ Column Kekre Transform } \\
\cline { 2 - 6 } & $\mathbf{( 1 6 , 1 6 )}$ & $\mathbf{( 3 2 , 8 )}$ & $\mathbf{( 8 , 3 2 )}$ & $\mathbf{( 6 4 , 4 )}$ & $\mathbf{( 4 , 6 4 )}$ & \\
\hline Resize4 & $\mathbf{3 5 . 9 1 4}$ & 38.134 & 36.762 & 38.913 & 38.020 & 38.538 \\
\hline Resize2 & $\mathbf{3 6 . 8 1 0}$ & 39.058 & 37.689 & 39.880 & 38.973 & 39.510 \\
\hline DFT_resize2 & 1.708 & 1.905 & $\mathbf{1 . 5 4 7}$ & 2.327 & 1.622 & 1.688 \\
\hline grid resize2 & 2.045 & 2.066 & $\mathbf{1 . 9 0 1}$ & 3.061 & 2.119 & 1.867 \\
\hline
\end{tabular}

Table 39. MAE values against resizing attacks when column DFT and column DFT wavelet are used to embed and extract watermark

\begin{tabular}{|c|c|c|c|c|c|c|}
\hline \multirow{2}{*}{ Resizing type } & \multicolumn{5}{|c|}{ Column RFT wavelet } & \multirow{2}{*}{ Column RFT } \\
\cline { 2 - 6 } & $\mathbf{( 1 6 , 1 6 )}$ & $\mathbf{( 3 2 , 8 )}$ & $\mathbf{( 8 , 3 2 )}$ & $\mathbf{( 6 4 , 4 )}$ & $\mathbf{( 4 , 6 4 )}$ & \\
\hline Resize4 & $\mathbf{2 7 . 7 4 6}$ & 29.338 & 28.752 & 31.383 & 29.305 & 30.610 \\
\hline Resize2 & $\mathbf{2 8 . 5 4 9}$ & 30.148 & 29.575 & 32.207 & 30.120 & 31.473 \\
\hline DFT_resize2 & 1.435 & 1.405 & 1.427 & $\mathbf{1 . 3 8 6}$ & 1.413 & 2.126 \\
\hline grid resize2 & 3.703 & 3.412 & 4.480 & $\mathbf{3 . 0 0 3}$ & 3.650 & 31.587 \\
\hline
\end{tabular}

Performance against resizing attack using orthogonal row transforms Haar, Walsh, Kekre Transform and Real Fourier Transform and corresponding row wavelet transforms in terms of MAE between embedded and extracted watermark is given in Table 40 to Table 43.

Table 40. MAE values against resizing attacks when row Haar and row Haar wavelet are used to embed and extract watermark

\begin{tabular}{|c|c|c|c|c|c|c|}
\hline \multirow{2}{*}{ Resizing type } & \multicolumn{5}{|c|}{ Row Haar wavelet } & \multirow{2}{*}{ Row Haar } \\
\cline { 2 - 6 } & $\mathbf{( 1 6 , 1 6 )}$ & $\mathbf{( 3 2 , 8 )}$ & $\mathbf{( 8 , 3 2 )}$ & $\mathbf{( 6 4 , 4 )}$ & $\mathbf{( 4 , 6 4 )}$ & \\
\hline Resize4 & 29.846 & 30.331 & 29.255 & $\mathbf{2 8 . 7 7 7}$ & 29.455 & 29.205 \\
\hline Resize2 & 30.665 & 31.156 & 30.058 & $\mathbf{2 9 . 5 7 3}$ & 30.257 & 30.013 \\
\hline DFT_resize2 & 1.177 & 1.338 & $\mathbf{1 . 0 7 7}$ & 1.094 & 1.188 & 1.225 \\
\hline grid resize2 & 3.583 & 4.028 & 3.919 & 3.864 & $\mathbf{3 . 3 9 6}$ & 4.106 \\
\hline
\end{tabular}

From Table 40, it can be observed that Row Haar wavelet transform performs marginally better than Row

Haar transform for each type of resizing attack.

Table 41. MAE values against resizing attacks when row Walsh and row Walsh wavelet are used to embed and extract watermark

\begin{tabular}{|c|c|c|c|c|c|c|}
\hline \multirow{2}{*}{ Resizing type } & \multicolumn{5}{|c|}{ Row Walsh wavelet } & \multirow{2}{*}{ Row Walsh } \\
\cline { 2 - 6 } & $\mathbf{( 1 6 , 1 6 )}$ & $\mathbf{( 3 2 , 8 )}$ & $\mathbf{( 8 , 3 2 )}$ & $\mathbf{( 6 4 , 4 )}$ & $\mathbf{( 4 , 6 4 )}$ & \\
\hline Resize4 & 31.345 & 32.915 & 31.941 & 33.634 & 33.633 & 30.878 \\
\hline Resize2 & 32.212 & 33.828 & 32.830 & 34.565 & 34.565 & 31.736 \\
\hline DFT_resize2 & $\mathbf{1 . 3 1 4}$ & 1.355 & 1.468 & 1.624 & 1.624 & 1.605 \\
\hline grid resize2 & 8.197 & $\mathbf{5 . 4 7 6}$ & 13.385 & 19.463 & 19.46299 & 42.759 \\
\hline
\end{tabular}

From Table 41, it can be concluded that for bicubic interpolation, Row Walsh is marginally better than Row Walsh wavelet transform. For DFT based resizing, Row Walsh wavelet obtained from $(16,16),(32,8)$ and $(8,32)$ combinations give marginally better MAE values and $(64,4)$ and $(4,64)$ combinations give almost equal MAE values and hence overall performance of Row Walsh wavelet can be considered acceptable over Row Walsh transform. For grid based resizing, significant improvement is observed by Row Walsh wavelet over Row Walsh transform. Similar observations are noted for row versions of Kekre Transform and Kekre wavelet 
Transform and also for row version of RFT and RFT wavelet transform.

Table 42. MAE values against resizing attacks when row Kekre Transform and row Kekre wavelet Transform are used to embed and extract watermark

\begin{tabular}{|c|c|c|c|c|c|c|}
\hline \multirow{2}{*}{ Resizing type } & \multicolumn{5}{|c|}{ Row Kekre wavelet } & \multirow{2}{*}{ Row Kekre Transform } \\
\cline { 2 - 6 } & $\mathbf{( 1 6 , 1 6 )}$ & $\mathbf{( 3 2 , 8 )}$ & $\mathbf{( 8 , 3 2 )}$ & $\mathbf{( 6 4 , 4 )}$ & $\mathbf{( 4 , 6 4 )}$ & \\
\hline Resize4 & 38.665 & $\mathbf{3 6 . 8 8 9}$ & 37.671 & 38.595 & 38.817 & 39.943 \\
\hline Resize2 & 39.627 & $\mathbf{3 7 . 8 1 0}$ & 38.608 & 39.547 & 39.781 & 40.953 \\
\hline FFT_resize2 & 1.795 & 1.828 & $\mathbf{1 . 5 8 1}$ & 2.119 & 1.735 & 1.605 \\
\hline grid resize2 & 2.097 & 2.479 & $\mathbf{1 . 9 9 7}$ & 2.764 & 2.479 & 2.183 \\
\hline
\end{tabular}

Table 43. MAE values against resizing attacks when row RFT and row RFT wavelet are used to embed and extract watermark

\begin{tabular}{|c|c|c|c|c|c|c|}
\hline \multirow{2}{*}{ Resizing type } & \multicolumn{5}{|c|}{ Row RFT wavelet } & \multirow{2}{*}{ Row RFT } \\
\cline { 2 - 6 } & $\mathbf{( 1 6 , 1 6 )}$ & $\mathbf{( 3 2 , 8 )}$ & $\mathbf{( 8 , 3 2})$ & $\mathbf{( 6 4 , 4 )}$ & $\mathbf{( 4 , 6 4 )}$ & \\
\hline Resize4 & 29.667 & 31.734 & 28.968 & 30.947 & 27.833 & 26.654 \\
\hline Resize2 & 30.486 & 32.606 & 29.779 & 31.794 & 28.629 & 27.397 \\
\hline FFT_resize2 & 1.604 & 1.430 & 1.660 & $\mathbf{1 . 2 2 6}$ & 2.032 & 2.382 \\
\hline grid resize2 & 3.890 & 3.563 & 5.430 & $\mathbf{2 . 1 7 0}$ & 9.638 & 30.373 \\
\hline
\end{tabular}

\section{COMPARISON OF WAVELET TRANSFORMS AGAINST} ATTACKS

In previous section, performance of orthogonal transform with their wavelet transform has been done. All those wavelet transforms are compared in this section.
For each attack, the best size combination of each wavelet transform is selected for comparison.

Fig. 7 below shows performance of Column DCT wavelet (CDW), Column Haar wavelet (CHW), Column Walsh wavelet (CWW), Column Kekre wavelet (CKW) and Column Real Fourier Wavelet (CRW) transforms against compression using DCT, Walsh, Haar and DST.

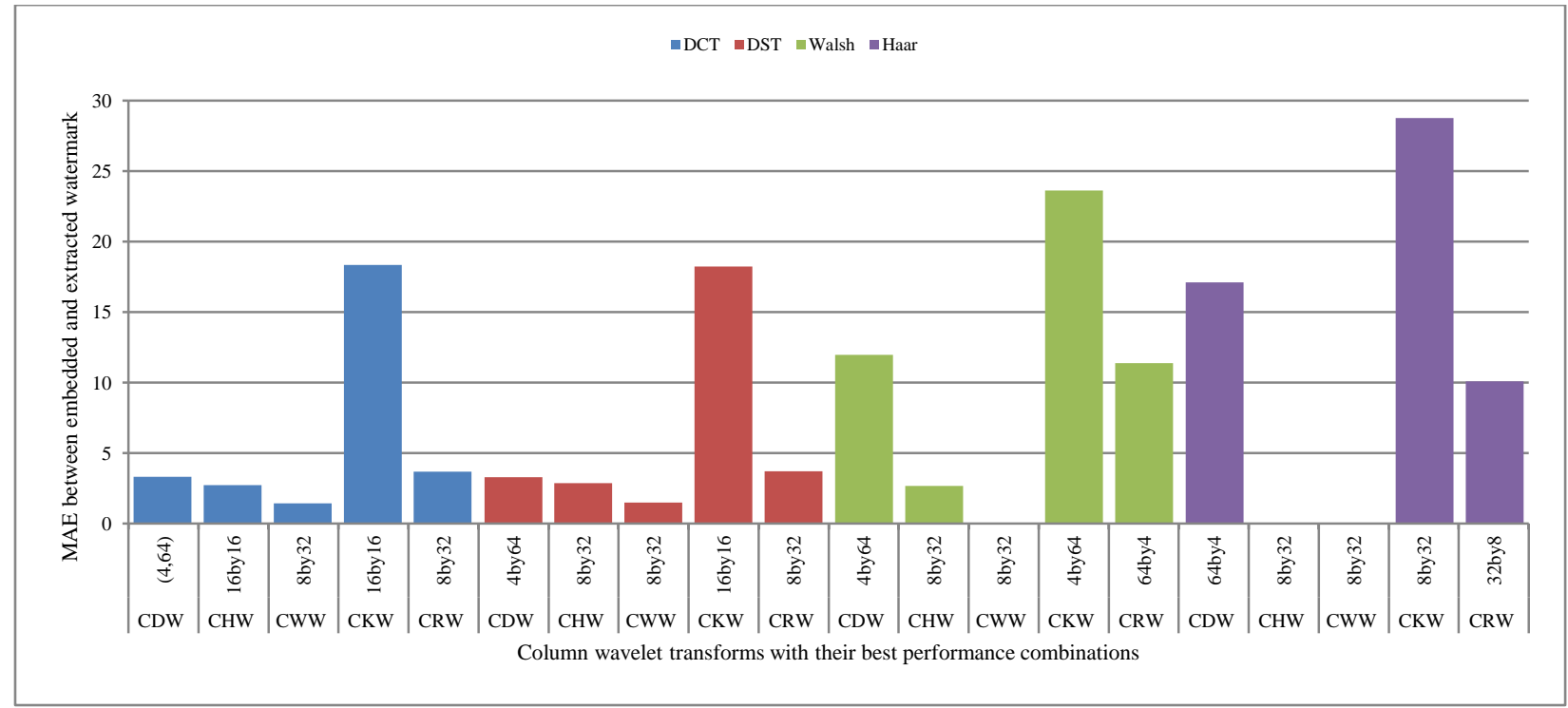

Fig. 7 Comparison of MAE between embedded and extracted watermark against compression using DCT, DST, Walsh and Haar when column DCT wavelet, Column Haar wavelet(CHW), Column Walsh wavelet(CWW), Column Kekre wavelet (CKW) and Column Real Fourier Wavelet (CRW) transforms are used for embedding watermark

From Fig. 7, column Walsh wavelet obtained from Walsh transform of size $8 \times 8$ and $32 \times 32$ is observed to be the best performer against compression using DCT, DST, Walsh and Haar. Column Haar wavelet transform closely follows it.
Fig. 8 shows the performance comparison of row DCT wavelet (RDW), row Haar wavelet (RHW), row Walsh wavelet (RWW), Row Kekre wavelet (RKW) and row Real Fourier wavelet transform (RRW) transforms against DCT, DST, Walsh and Haar based compression. 


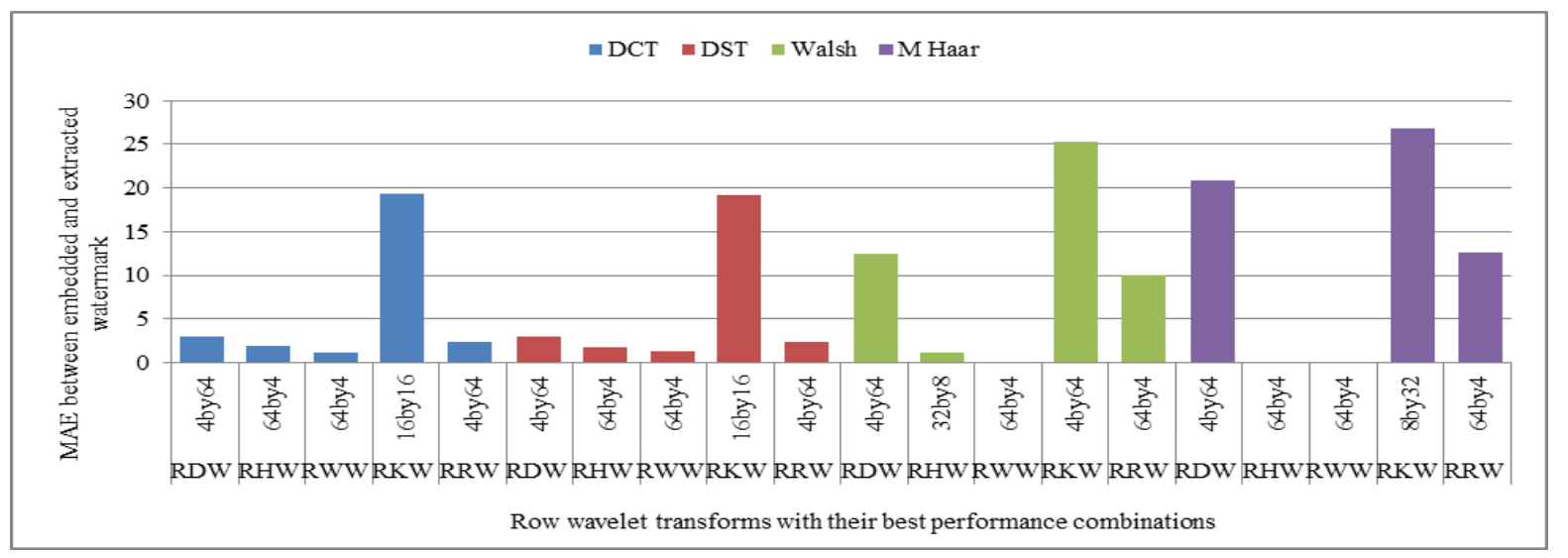

Fig. 8. Comparison of MAE between embedded and extracted watermark against compression using DCT, DST, Walsh and Haar when row DCT wavelet (RDW), row Haar wavelet(RHW), row Walsh wavelet(RWW), row Kekre wavelet (RKW) and row Real Fourier Wavelet (RRW) tran sforms are used for embedding watermark

Row Walsh wavelet closely followed by row Haar wavelet transform are most robust against compression using DCT, DST, Walsh and Haar as can be observed from Fig. 8 .
Fig. 9 and Fig. 10 show comparison of column transforms and row transforms respectively against compression using DCT wavelet, JPEG compression and VQ based compression.

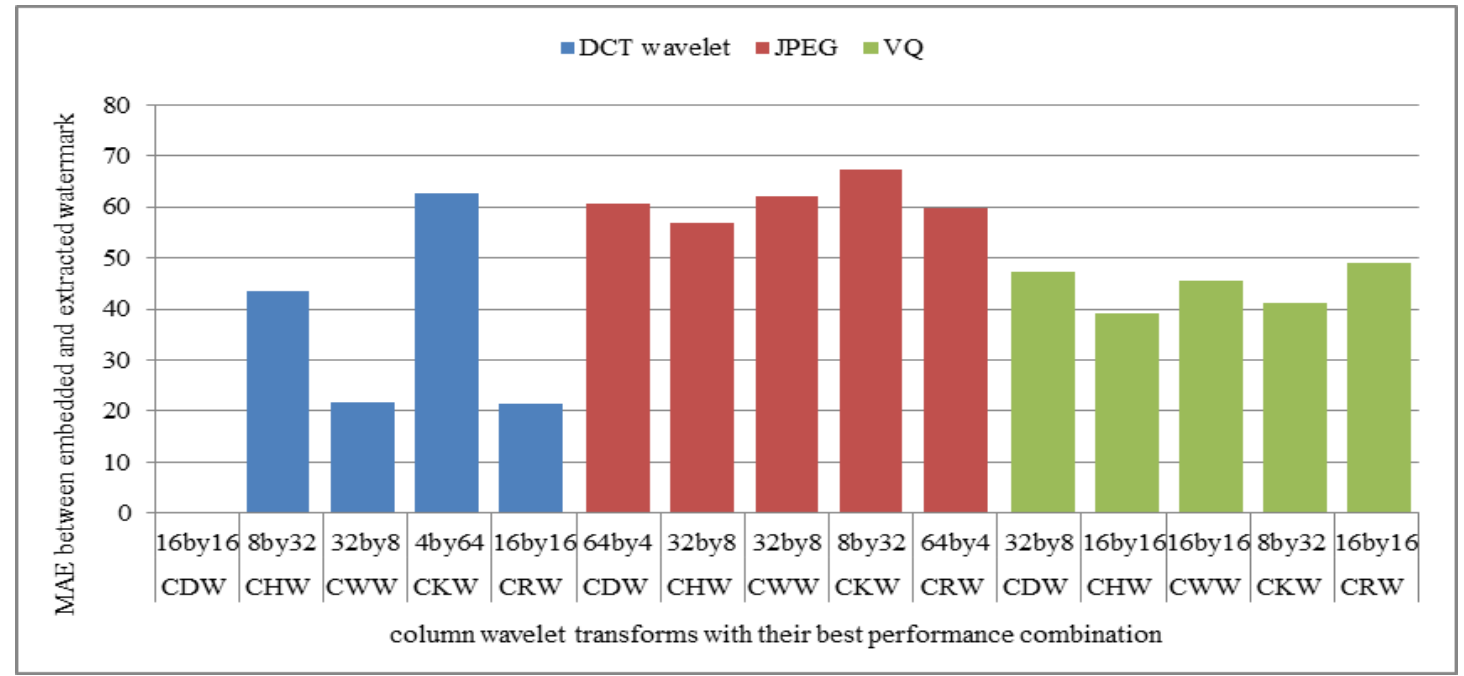

Fig. 9. Comparison of MAE between embedded and extracted watermark against compression using DCT wavelet, JPEG and VQ when column DCT wavelet, Column Haar wavelet(CHW), Column Walsh wavelet(CWW), Column Kekre wavelet (CKW) and Column Real Fourier Wavelet (CRW) transforms are used for embedding watermark

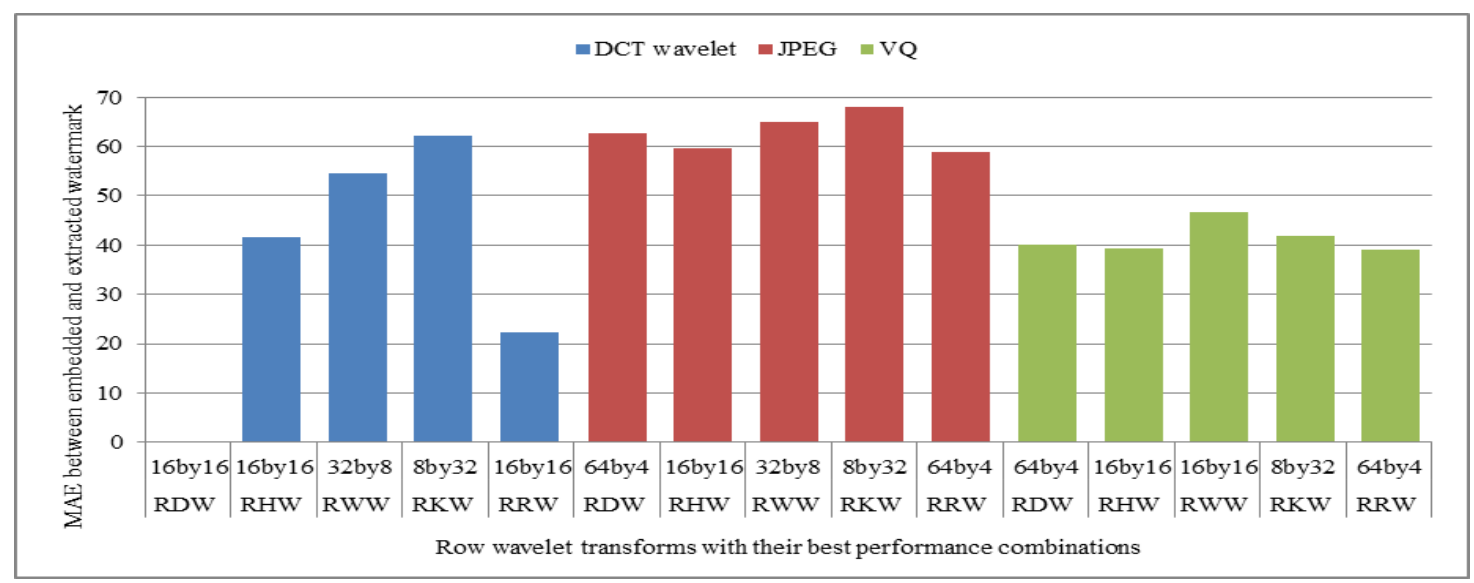

Fig. 10. Comparison of MAE between embedded and extracted watermark against compression using DCT wavelet, JPEG and VQ when row DCT wavelet (RDW), row Haar wavelet(RHW), row Walsh wavelet(RWW), row Kekre wavelet (RKW) and row Real Fourier Wavelet (RRW) tran sforms are used for embedding watermark 
From Fig. 9 and 10 it can be seen that for compression using column DCT wavelet, column DCT wavelet and row DCT wavelet obtained from 16x16 size DCT matrix give best robustness with MAE zero. For JPEG compression column Haar wavelet $(32,8)$ and row Real Fourier wavelet $(64,4)$ are better than other column and row wavelet transforms. For VQ based compression, column and row Haar wavelet obtained from $(16,16)$ size Haar matrix are better.

Comparison of column wavelet transforms and row wavelet transforms against cropping attack is shown in Fig. 11 and Fig. 12 respectively.

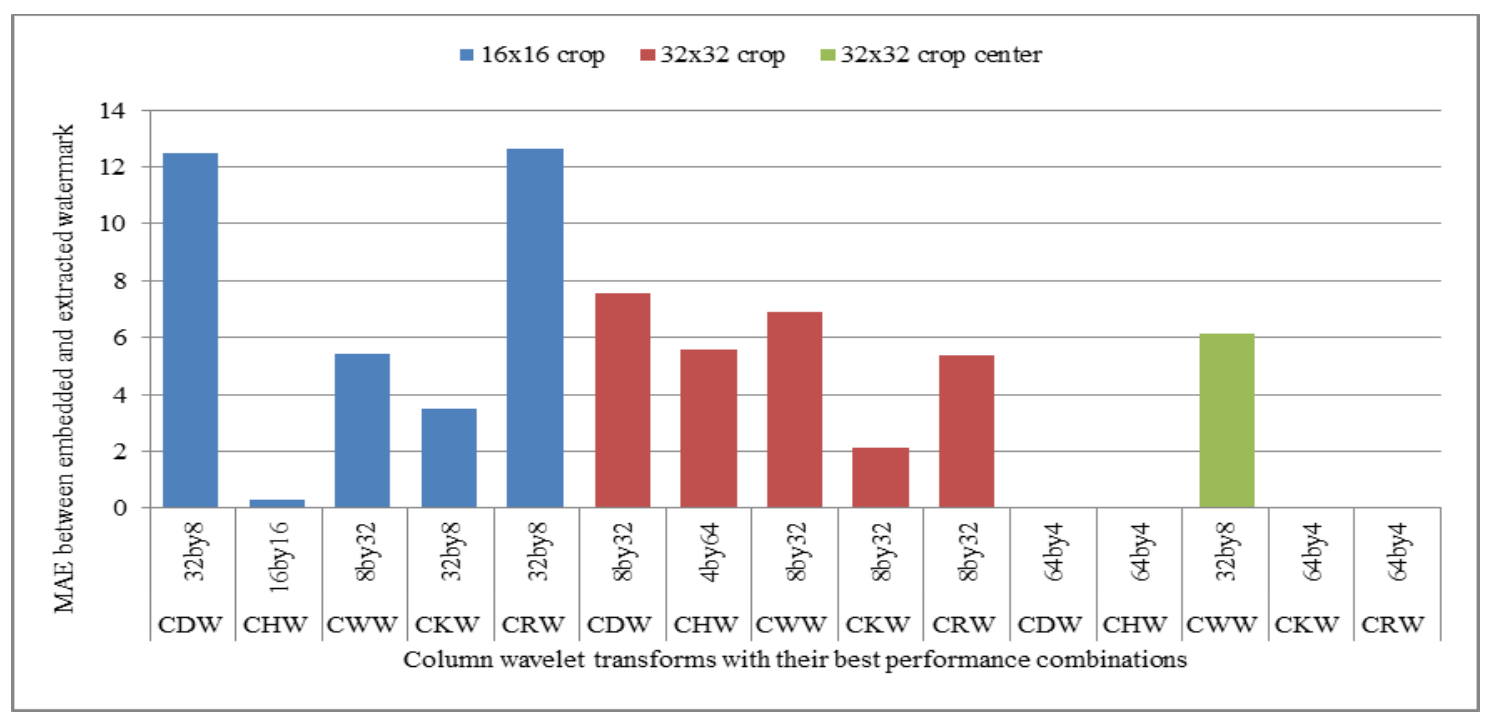

Fig. 11. Comparison of MAE between embedded and extracted watermark against cropping when column DCT wavelet, Column Haar wavelet(CHW), Column Walsh wavelet(CWW), Column Kekre wavelet (CKW) and Column Real Fourier Wavelet (CRW) transforms are used for embedding watermark

From Fig. 11, it is observed that as we increase amount of cropped portion at corners, MAE between embedded and extracted watermark is reduced except for column Haar transform. For $16 \times 16$ cropping, column Haar wavelet transform and for $32 \times 32$ cropping at corners, column Kekre wavelet transform are observed to be robust than others. For 32x32 cropping at center, except
Walsh column wavelet all other column wavelet transforms show outstanding robustness with MAE zero. This is applicable for row wavelet transforms shown in Fig. 12. Row DCT wavelet and row Kekre wavelet are more robust against $16 \times 16$ and 32×32 cropping at corners respectively.

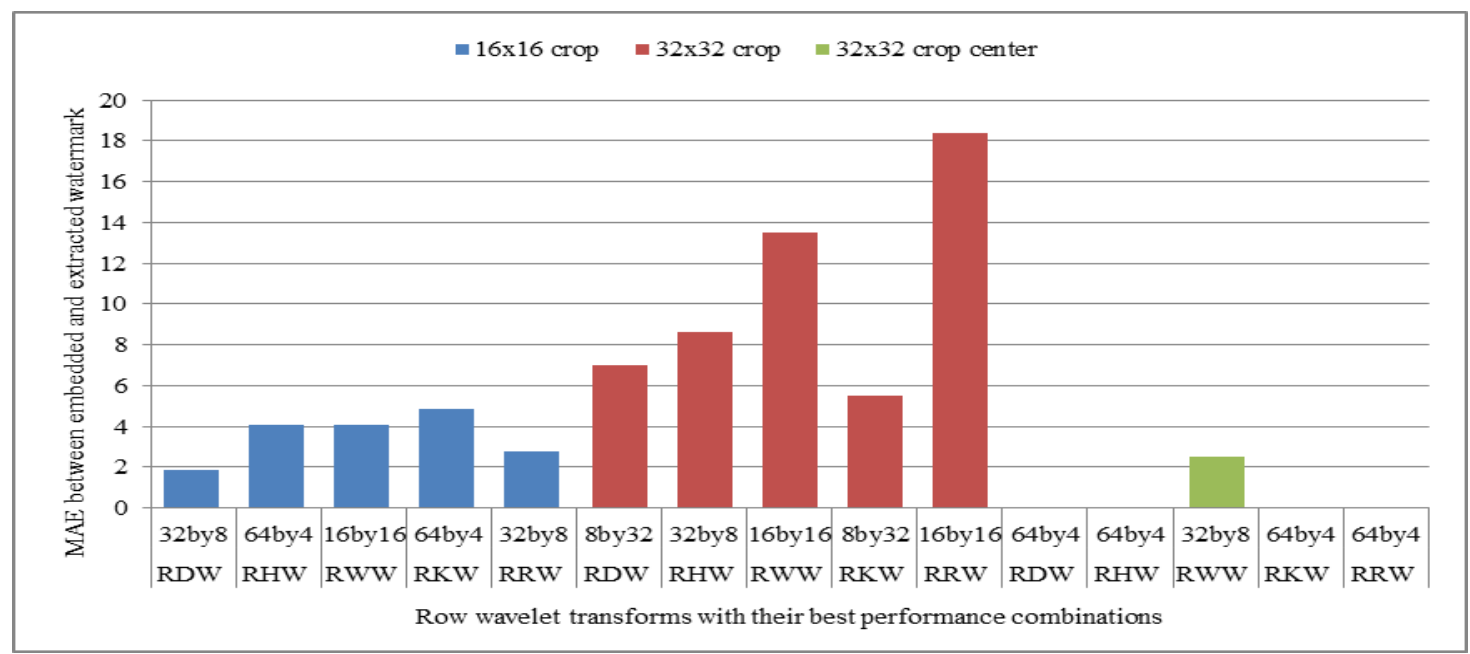

Fig. 12. Comparison of MAE between embedded and extracted watermark against cropping when row DCT wavelet (RDW), row Haar wavelet(RHW), row Walsh wavelet(RWW), row Kekre wavelet (RKW) and row Real Fourier Wavelet (RRW) transforms are used for embedding watermark

Fig. 13 and Fig. 14 show performance comparison of column wavelets and row wavelets respectively against noise addition attack. All column transforms show zero MAE against Binary distributed run length noise with run length 1 to 10 and hence not shown in Fig. 13. For higher run length of binary distributed run length noise and for Gaussian distributed run length noise, column Kekre wavelet is observed to be most robust. 


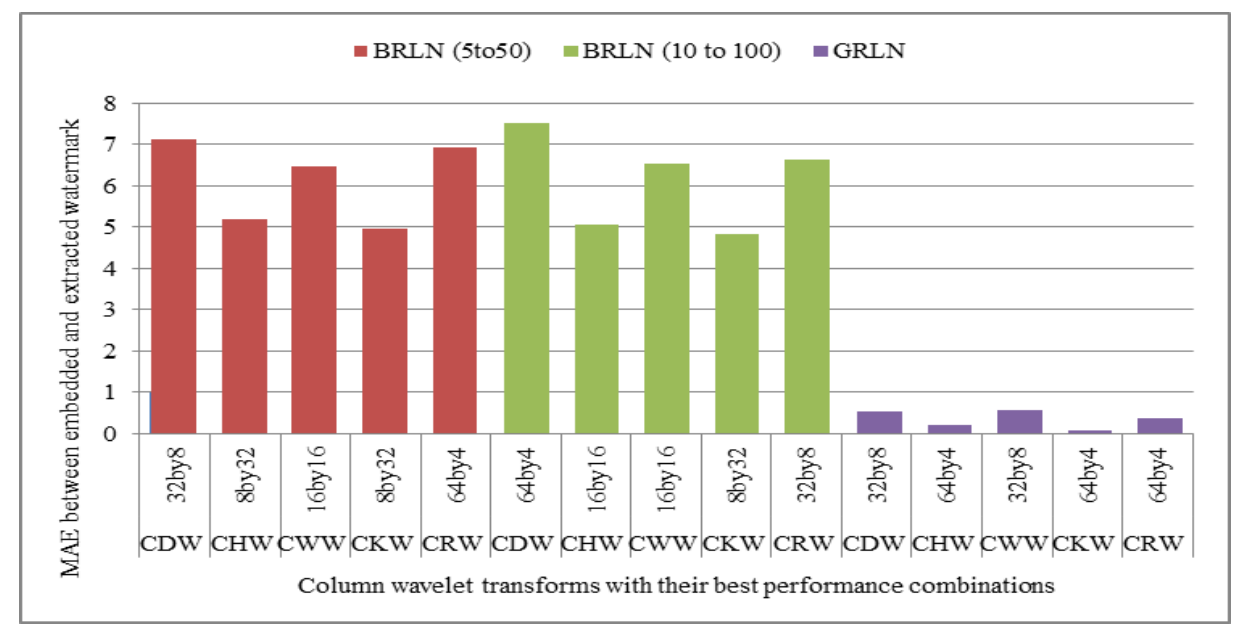

Fig. 13. Comparison of MAE between embedded and extracted watermark against noise addition when column DCT wavelet, Column Haar wavelet(CHW), Column Walsh wavelet(CWW), Column Kekre wavelet (CKW) and Column Real Fourier Wavelet (CRW) transforms are used for embedding watermark

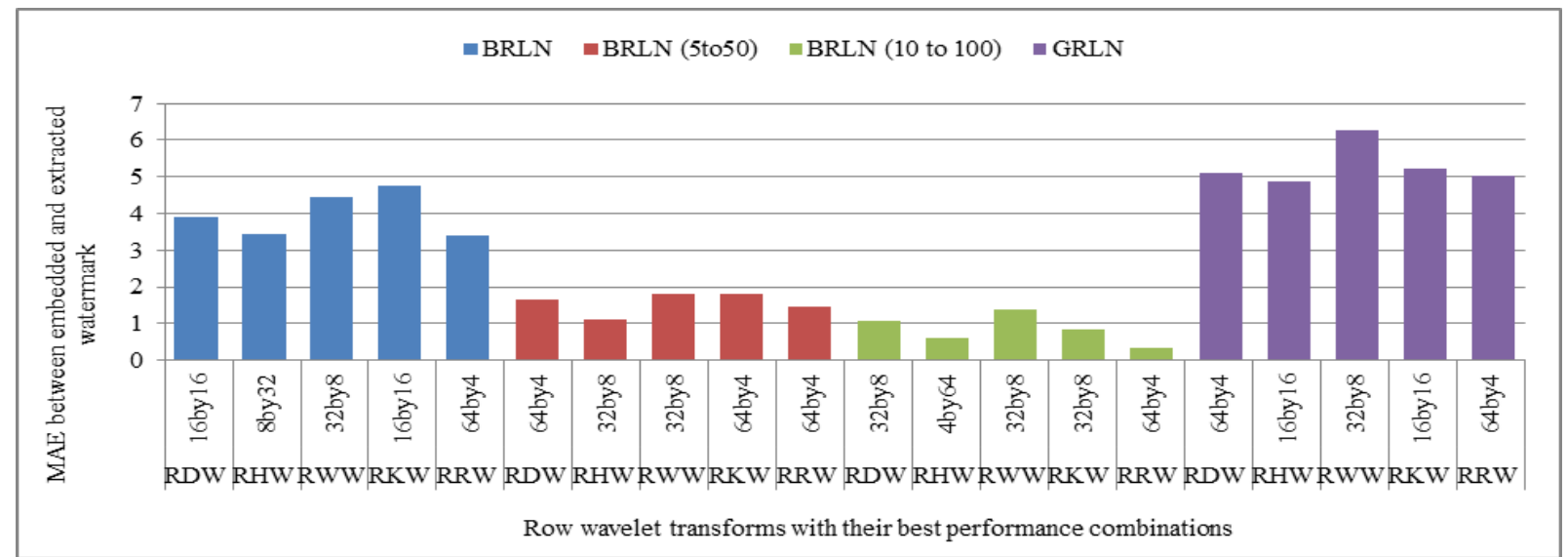

Fig. 14. Comparison of MAE between embedded and extracted watermark against noise addition when row DCT wavelet (RDW), row Haar wavelet(RHW), row Walsh wavelet(RWW), row Kekre wavelet (RKW) and row Real Fourier Wavelet (RRW) transforms are used for embedding watermark

From Fig. 14, it is observed that for all varieties of noise addition attack, except binary distributed run length noise with 10 to 100 run length Haar wavelet is better in robustness. Real Fourier row wavelet transform is slightly better than Row Haar wavelet for 10 to 100 run length of binary distributed run length noise

Fig. 15 shows comparison of column wavelet transforms and Fig. 16 shows comparison of row wavelet transforms against various resizing attacks.

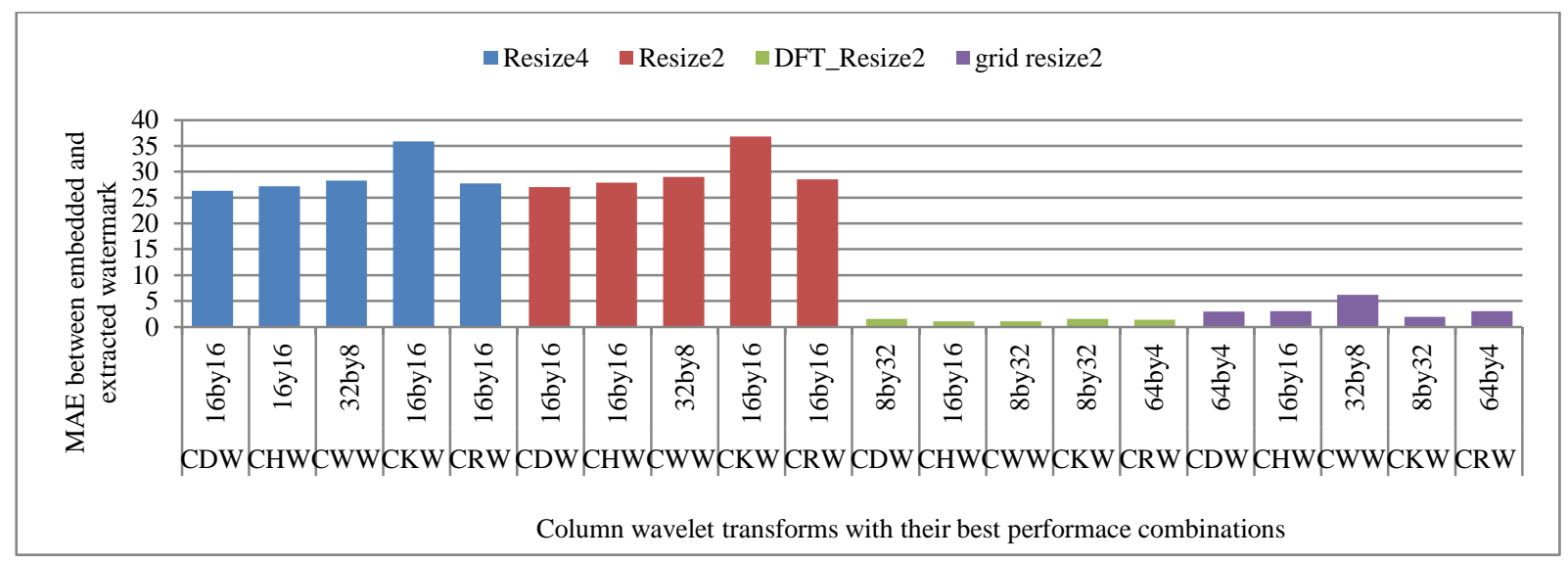

Fig. 15. Comparison of MAE between embedded and extracted watermark against resizing attack when column DCT wavelet, Column Haar wavelet(CHW), Column Walsh wavelet(CWW), Column Kekre wavelet (CKW) and Column Real Fourier Wavelet (CRW) transforms are used for embedding watermark 


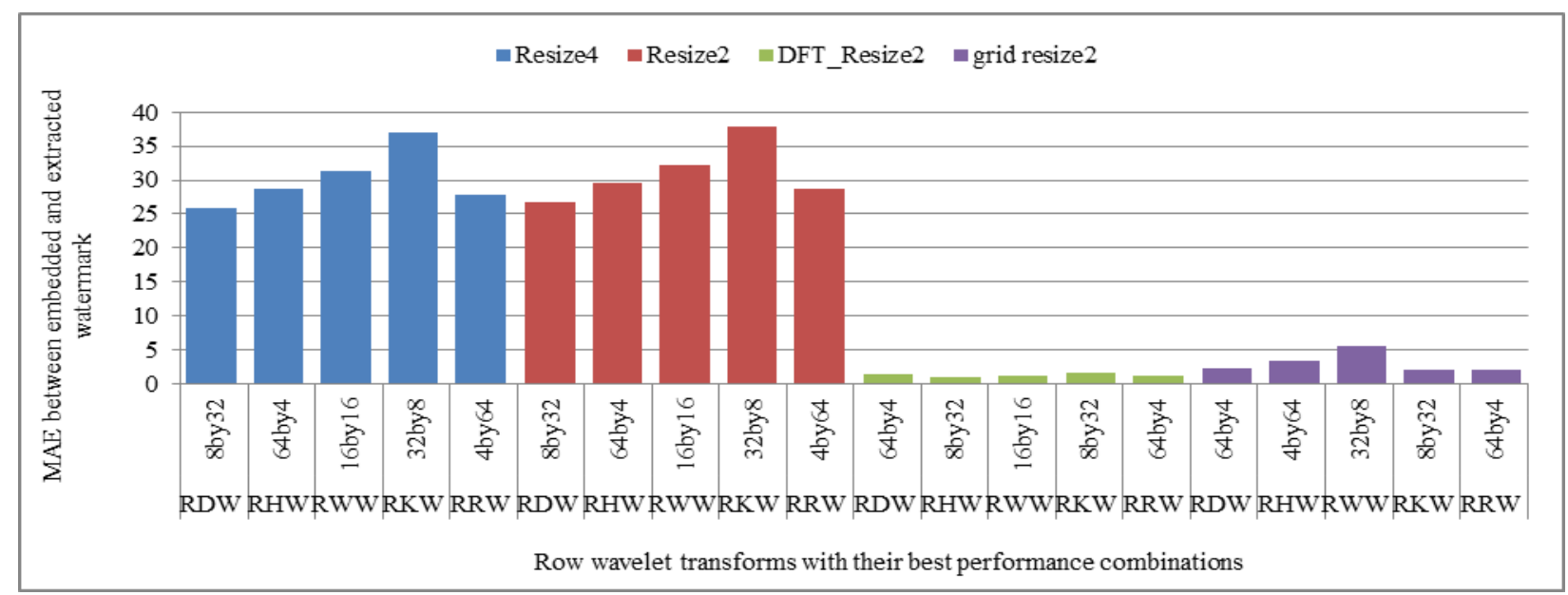

Fig. 16. Comparison of MAE between embedded and extracted watermark against resizing attack when row DCT wavelet (RDW), row Haar wavelet(RHW), row Walsh wavelet(RWW), row Kekre wavelet (RKW) and row Real Fourier Wavelet (RRW) transforms are used for embedding watermark

From Fig. 15 and 16 we can observe that column and row DCT wavelet show better robustness than other column and row transforms against resizing using bicubic interpolation which is named as Resize 4 and Resize 2 in graphs. For DFT based resizing all column wavelet transforms as well as row wavelet transforms show negligible difference in performance. For grid based resizing, column and row Kekre wavelet transforms are better than other column and row wavelet transforms respectively.

\section{CONCLUSION}

The proposed method uses orthogonal transforms DCT, Haar, Walsh, Kekre transform and Real Fourier Transform and their wavelets generated using different size combinations of these orthogonal transforms. Wavelet transforms when used with SVD for embedding watermark give better robustness than orthogonal transforms with SVD. For different attacks, different size combination of orthogonal transform used to generate wavelet transform gives best robustness and the overall performance of wavelet transforms is better than orthogonal transform in column and row version. Wavelet transforms when compared, different wavelets are found to be good in robustness against different attacks.

\section{REFERENCES}

[1] Veysel Aslantas, A. Latif Dogan and Serkan Ozturk, "DWT-SVD based image watermarking using particle swarm optimizer", Proc. of IEEE International Conference on Multimedia and Expo, pp. 241-244. 2008.

[2] Yang Quianli, Cai Yanhong, "A digital watermarking algorithm based on DWT and DCT", IEEE International Symposium on Information Technology in Medicine and Education, pp. 1102-1105, 2012.

[3] Xi-Ping he and Qing-Sheng Zhu, "A robust waveletdomain watermarking algorithm for colour image", Proceedings of the fifth international conference on machine learning and cybernetics, Dalian, pp.13-16, August 2006.
[4] Zhen Li, Kim-Hui Yap and Bai-Ying Lei, "A new blind robust image watermarking scheme in SVD-DCT composite domain", In Proc. of 18th IEEE international conference on Image Processing, pp. 2757-2760, 2011.

[5] Rahim Ansari, Mrutyunjaya M Devanalamath, K. Manikantan, S. Ramachandran, "Robust digital image watermarking algorithm in DWT-DFT-SVD domain for colour images", Proc. of IEEE International Conference on Communication, Information \& Computing Technology (ICCICT), Oct. 19-20 2012, Mumbai, India, pp. 1-6, Oct 2012.

[6] Zhen Li, Kim-Hui Yap and Bai-Ying Lei, "A new blind robust image watermarking scheme in SVD-DCT composite Domain", 18th IEEE International Conference on Image Processing, 2011, pp. 2757-2760, 1987.

[7] Yan Dejun, Yang Rijing, Li Hongyan, and Zheng Jiangchao, "A Digital Watermarking Scheme Based On Singular Value Decomposition and Discrete Wavelet Transform", In IEEE Proc. of International Conference on Computer Science and Network Technology, pp. 154-157, 2011.

[8] Krzysztof Simek, "Properties of SVD based dynamical model of gene expressing data", International Journal of Applied Maths Computer Science, Vol. 13, No. 3, pp. 337-345, 2003.G.Wyszecki and W.Stiles, Color Science: Concepts and Metheds, Quantitative Data and Formulae, 2nd ed. New York: Wiley, 1982.

[9] Cao, Lijie, "Singular value decomposition applied to digital image processing", Division of Computing Studies, Arizona State University Polytechnic Campus, Mesa, 2006.

[10] Ruizhen Liu, Tieniu Tan, "A SVD based watermarking scheme for protecting rightful ownership", IEEE transactions on multimedia, vol. 4, pp. 121-128, March 2002.

[11] Kekre, H. B., Archana Athawale, Dipali Sadavarti, "Algorithm to Generate Wavelet Transform from an Orthogonal Transform.” International Journal of Image Processing (IJIP) 4.4 (2010): 444

[12] H. B. Kekre, Tanuja Sarode, Sudeep Thepade. "Grid based image scaling technique", International Journal of Computer Science and Applications, Volume 1, No. 2, pp. 95-98, August 2008.

[13] I. J. Cox, J. Killian, F. T. Leighton, T. Shamoon, "Secure spread spectrum watermarking for multimedia", IEEE Transaction on Image Processing, 6(12), pp. 1673-1687, 
1997.

[14] Dr. H. B. Kekre, Dr. Tanuja Sarode, Shachi Natu. "Image zooming using Sinusoidal Transforms like Hartley, DFT, DCT, DST and Real Fourier Transform", selected for publication in International journal of computer science and information security Vol. 12 No. 7, July 2014.

[15] H. B. Kekre, Tanuja Sarode, Sudeep Thepade, "Inception of Hybrid Wavelet Transform using Two Orthogonal Transforms and It's use for Image Compression", International Journal of Computer Science and Information Security, volume 9, No. 6, pp. 80-87, 2011.

\section{Authors' Profiles}

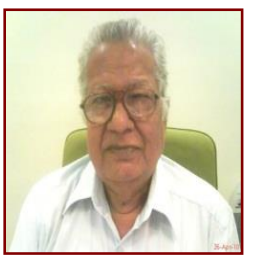

Dr. H. B. Kekre has received B.E. (Hons.) in Telecomm. Engg. from Jabalpur University in 1958, M.Tech (Industrial Electronics) from IIT Bombay in 1960, M.S.Engg. (Electrical Engg.) from University of Ottawa in 1965 and Ph.D. (System Identification) from IIT Bombay in 1970. He has worked Over 35 years as Faculty of Electrical Engineering and then HOD Computer Science and Engg. at IIT Bombay. After serving IIT for 35 years, he retired in 1995. After retirement from IIT, for 13 years he was working as a professor and head in the department of computer engineering and Vice principal at Thadomal Shahani Engg. College, Mumbai. Now he is senior professor at MPSTME, SVKM's NMIMS University. He has guided 17 Ph.Ds., more than 100 M.E./M.Tech and several B.E. / B.Tech projects, while in IIT and TSEC. His areas of interest are Digital Signal processing, Image Processing and Computer Networking. He has more than 450 papers in National / International Journals and Conferences to his credit. He was Senior Member of IEEE. Presently He is Fellow of IETE, Life Member of ISTE and Senior Member of
International Association of Computer Science and Information Technology (IACSIT). Recently fifteen students working under his guidance have received best paper awards.

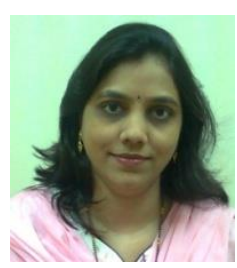

Dr. Tanuja K. Sarode has received M.E. (Computer Engineering) degree from Mumbai University in 2004, Ph.D. from Mukesh Patel School of Technology, Management and Engg. SVKM's NMIMS University, Vile-Parle (W), Mumbai, INDIA. She has more than 14 years of experience in teaching. Currently working as Associate Professor in Dept. of Computer Engineering at Thadomal Shahani Engineering College, Mumbai. She is member of International Association of Engineers (IAENG) and International Association of Computer Science and Information Technology (IACSIT). Her areas of interest are Image Processing, Signal Processing and Computer Graphics. She has more than 150 papers in National /International Conferences/journal to her credit.

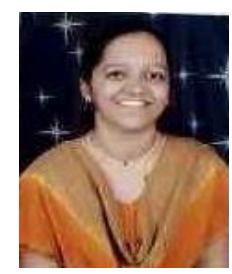

Ms. Shachi Natu has received M.E. (Computer Engineering) degree from Mumbai University in 2010. Currently pursuing Ph.D. from NMIMS University. She has 10 years of experience in teaching. Currently working as Assistant Professor in Department of I.T. at Thadomal Shahani Engineering College, Mumbai. Her areas of interest are Image Processing, Database Management Systems and Operating Systems. She has 27 papers in International Conferences/journal to her credit.

How to cite this paper: H. B. Kekre, Tanuja Sarode, Shachi Natu,"Performance Comparison of Watermarking Using SVD with Orthogonal Transforms and Their Wavelet Transforms", IJIGSP, vol.7, no.4, pp.1-18, 2015.DOI: 10.5815/ijigsp.2015.04.01 